\title{
Spider Diagrams with Absence: Inference Rules for Clutter Reduction
}

\author{
Gem Stapleton ${ }^{1}$ and Lopamudra Choudhury ${ }^{2}$ and Mihir Chakraborty ${ }^{2}$ \\ ${ }^{1}$ Centre for Secure, Intelligent and Usable Systems, \\ University of Brighton, UK \\ 2 Jadavpur University, India \\ g.e.stapleton@brighton.ac.uk, choudhuryl@yahoo.com, mihirc4@gmail.com
}

\begin{abstract}
Spider diagrams represent sets, their cardinalities and, sometimes, the specific individuals within those sets. They are expressively equivalent to monadic first-order logic with equality. Typically, diagrammatic logics with this level of expressiveness are not equipped to directly express the absence of an individual from a set. Instead, individuals must be asserted to be present and, thus, absent from the set's complement. The first time that absence could be directly asserted was in Venn-i. Since then, it been shown that in a related system called Venn- $i^{e}$ (a monadic first-order logic without equality) the inclusion of absence information can significantly reduce diagram clutter. In this paper, we explore an extension of spider diagrams to include direct representation of the absence of individuals from sets. We identify necessary and sufficient conditions for satisfiability, allowing us to define an inconsistency rule allowing significant reductions in diagram clutter. Building on that, we introduce sound inference rules specifically related to spiders (which represent elements, individuals or their $a b$ sence) that alter the levels of clutter in consistent diagrams. In the context of these rules, we explore the implications of including absence information for reducing clutter. In particular, we show that the significant benefits, in terms of clutter reduction, seen through the use of absence in Venn-i ${ }^{e}$ do not manifest to such an extent in spider diagrams.
\end{abstract}

\section{Introduction}

The ability to negate statements plays a crucial role in all logics. The notion of absence is closely related to that of negation: $a \notin P$ (i.e. the individual $a$ is not in the set $P$ ) indicates, informally speaking, that the individual $a$ is $a b$ sent from $P$. Indeed, the importance of negation should not be underestimated, "The capacity to negate is the capacity

DOI reference number: 10.18293/VLSS2018-032.

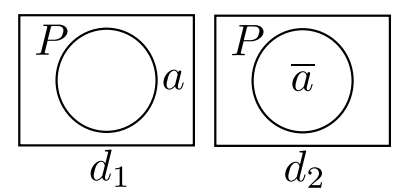

Figure 1. Asserting presence and absence.

to refuse, to contradict, to lie, to speak ironically, to distinguish truth from falsity - in short, the capacity to be human" [7]. In diagrams research, though, it has long been believed that diagrams are not well equipped to make negated statements directly. Indeed, even simple statements like $a \notin P$ cannot be made explicitly in most Euler diagrambased logics, such as $[10,14,18,19]$. Instead, these types of diagrams tend to assert $a \in \bar{P}$ (the complement of $P$ ).

There is an exception to this: Choudhury and Chakraborty developed a diagrammatic logic named Venn$\mathrm{i}$ that allows $a \notin P$ to be directly expressed [4]. The Venn-i logic builds on Shin's Venn-I system [15], which exploits Peirce's $\otimes$-sequences to indicate the non-emptiness of sets [13]. Venn-i also uses $i$-sequences and $\bar{i}$-sequences to represent individuals and, respectively, their absence. Choudhury and Chakraborty adopt a classical interpretation, meaning that the absence of an individual from one set implies its presence in the complement ${ }^{1}$. An inspiration for Choudhury's and Chakraborty's work came from the notion of abhāva (absence). Abhāva, an important feature of ancient Indian knowledge systems, allocates a first class status to the absence of individuals.

Examples can be seen in figure 1 . The diagram $d_{1}$ directly expresses that $a$ is in $\bar{P}$, since the location of the symbol $a$ is outside the curve $P$. From this, we can deduce that $a$ is not in $P$, that is, $a$ is absent from $P$. By contrast,

\footnotetext{
${ }^{1}$ A related system, developed by Bhattacharjee et al. focuses on a nonclassical interpretation of absence [2]. In that system, the absence of an individual from one set does not imply its presence in the complement. They devised a sound and complete set of inference rules which allow diagrammatic proofs to be written when absence information is given.
} 


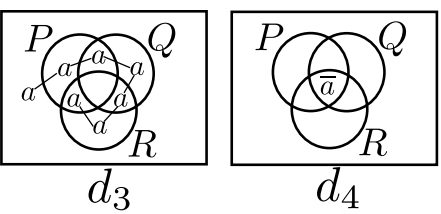

Figure 2. Clutter reduction.

the diagram $d_{2}$ directly expresses that $a$ is absent from $P$ by placing $\bar{a}$ inside $P$. Thus, the use of ${ }^{-}$depicts the absence of individuals from a set. Whilst this example is sufficient to illustrate the use of absence, it does not demonstrate the role that absence can play in reducing diagram clutter. We explore this more fully later in the paper. For now, suppose we want to express that $a$ is absent from $P \cap Q \cap R$. Referring to figure 2 , this is achieved indirectly by $d_{3}$ which expresses that $a \in \overline{P \cap Q \cap R}$ (i.e. the complement of $P \cap Q \cap R$ ). The diagram $d_{4}$ is semantically equivalent but instead expresses the required statement, $a \notin P \cap Q \cap R$, directly using absence. The diagram $d_{4}$ is arguably less cluttered than $d_{3}$.

Clutter in Euler diagrams was studied by John et al. [12]: they devised a theoretical measure of clutter. Alqadah et al. empirically found that increasing levels of clutter in Euler diagrams negatively impacts user task performance [1]. Whilst empirically studying concept diagrams [11] (which extend Euler diagrams with syntax for individuals amongst other things), Hou et al. found that the diagrams where people had trouble performing tasks were those with the higher levels of clutter [8,9]. Hence, there is clearly a need to be able to measure the level clutter in diagrams generally and its impact on end-user task performance.

In previous work we, with Burton, demonstrated that explicitly representing the absence of individuals allows information to be presented in a less cluttered way [3]. This was in an Euler diagram system, called Venn- $\mathrm{i}^{\mathrm{e}}$, that incorporated $\otimes$-sequences, $i$-sequences (like the $a$-sequence in $d_{3}$ ) and $\bar{i}$-sequences (like $\bar{a}$ in $d_{4}$ ). We note here that, just as in Venn-i, distinct $i$-sequences need not represent distinct individuals. As such, Venn- $\mathrm{i}^{\mathrm{e}}$ is a monadic first-order logic (without equality). An empirical evaluation suggested, for Venn- $\mathrm{i}^{\mathrm{e}}$, high levels of clutter arising from individuals is detrimental to human cognition [16].

There are two key points: (a) rising levels of diagram clutter negatively impacts human cognition and (b) representing absence directly in systems with the expressiveness of monadic first-order logic (without equality) can bring reductions in clutter. An obvious question arises: does the inclusion of absence in more expressive systems still bring with it possibility of reducing clutter to the same extent? This paper helps us to understand clutter arising from spiders (akin to sequences) in an extended version of spider diagrams, which we call enhanced spider diagrams. This increases the level of expressiveness, over which we can explore the role of absence, to monadic first-order logic with equality. In particular, we make the following contributions:

- identify how to extend spider diagrams to include absence information (section 2).

- formalize the syntax and semantics of enhanced spider diagrams (section 3),

- identify necessary and sufficient conditions for enhanced spider diagrams to be unsatisfiable (section 4),

- introduce inference rules for enhanced spider diagrams that apply specifically to spiders (section 5),

- discuss how the inference rules can be used to alter the level of clutter present in enhanced spider diagrams (section 6), and

- draw comparison to the Venn-i $\mathrm{i}^{\mathrm{e}}$ case in section 7.

We conclude and identify future work in section 8 . This paper directly extends [17].

\section{Representing Absence in Spider Diagrams}

We now proceed to, briefly, show how absence can be incorporated into spider diagrams [5], which typically include existential spiders for denoting the existence of elements in sets. In addition, they have been studied with the inclusion of constant spiders [18] which, in this paper, we refer to as positive spiders. These spiders represent the presence of specific individuals in particular sets.

An example can be seen in figure 3 . The spider diagram $d_{5}$ expresses the following: (i) due to the spatial relationships between the curves, $R$ is disjoint from $P$ and from $Q$; (ii) due to the inclusion of two spiders, there are at least two elements, one of which (denoted by the existential spider comprising two nodes) is in $P$ and the other of which (denoted by the positive spider $a$ ) is the individual $a$ and is in the set $P \backslash Q$, and (iii) due to the shading, combined with the existential spider, there is at most one element in $P \cap Q$.

The diagram $d_{6}$ augments $d_{5}$ with additional information expressed by four negative spiders: (iv) the individual $b$ is not in $\bar{P}$ and the individual $c$ is not in $R$. Therefore, from (iv), $b$ is in the set $P$. An obvious question then arises: should the individual $b$ be necessarily different from the two elements represented by the existential spider and the positive spider $a$ ? In our view, the most diagrammatic interpretation is that $b$ is not necessarily different. It seems natural to say that two existential or positive spiders placed in a common region represent distinct individuals since they are represented by distinct syntactic devices. However, it does not seem very diagrammatic for $d_{6}$ to force $P$ to contain at least three elements when we can see only two spiders inside $P$. This observation suggests that the diagram would not be well-matched to its semantics [6] if we forced $b$ to 
denote the presence of an additional element in $P$. Therefore, in our extension of spider diagrams to include absence information directly, via negative spiders, we do not interpret negative spiders as providing distinctness information about individuals, unlike existential and positive spiders.

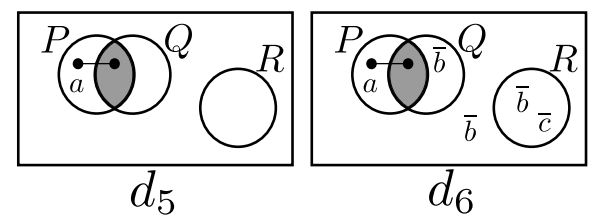

Figure 3. Incorporating absence.

\section{Syntax and Semantics}

Having introduced how absence can be incorporated into spider diagrams, we now formally define the syntax and semantics of the enhanced system. It is helpful for us to have a countably infinite set of labels from which all labels used on the curves in any diagram are drawn; we call this set $\mathcal{L}$. A zone is a pair, (in, out), where in and out are finite, disjoint subsets of $\mathcal{L}$. Given $\mathcal{L}$, the set of all zones is denoted $\mathcal{Z}$. In figure 3 , each diagram has five zones, such as the one inside both $P$ and $Q$ but outside $R$; this zone is $(\{P, Q\},\{R\})$. We also have a countably infinite set of constant symbols, denoted $\mathcal{C}$, which are used as labels for positive and negative spiders. So, in figure 3, spider labels $a, b$ and $c$ appear. Using these predefined sets, we can now formally define an enhanced spider diagram at the abstract syntax level:

Definition 1. An enhanced spider diagram, $d$, is a tuple, $d=(L, Z, S h Z, E S, P S, N S, \eta, \rho)$ such that:

1. L is a finite set of labels chosen from $\mathcal{L}$.

2. $Z$ is a set of zones where $(\emptyset, L) \in Z$ and for all (in, out) in $Z$, in $\cup$ out $=L$.

3. Sh $Z$ is a subset of $Z$ whose elements are called shaded zones.

4. ES, PS, NS are finite pairwise disjoint sets whose elements are called existential spiders, positive spiders, and negative spiders respectively.

5. $\eta: E S \cup P S \cup N S \rightarrow \mathbb{P} Z \backslash\{\emptyset\}$ returns the location of each spider.

6. $\rho: P S \cup N S \rightarrow \mathcal{C}$ returns the label of each positive and negative spider.

We further define $S(d)=E S \cup P S \cup N S$ to be the set of spiders in $d$.

In figure $3, d_{6}$ has the following abstract syntax:

1. label set $L=\{P, Q, R\}$,
2. zone set $Z=\{(\{P\},\{Q, R\}),(\{Q\},\{P, R\})$, $(\{P, Q\},\{R\}),(\{R\},\{P, Q\}),(\emptyset,\{P, Q, R\})\}$,

3. shaded zone set $S h Z=\{(\{P, Q\},\{R\})\}$,

4. existential spider set $E S=\left\{\sigma_{1}\right\}$, positive spider set $P S=\left\{\sigma_{a}\right\}$, and negative spider set $N S=$ $\left\{\sigma_{b, 1}, \sigma_{b, 2}, \sigma_{b, 3}, \sigma_{c}\right\}$,

5. spider locations given by

$$
\begin{aligned}
\eta\left(\sigma_{1}\right) & =\{(\{P\},\{Q, R\}),(\{P, Q\},\{R\})\}, \\
\eta\left(\sigma_{a}\right) & =\{(\{P\},\{Q, R\}), \\
\eta\left(\sigma_{b, 1}\right) & =\{(\{Q\},\{P, R\})\}, \\
\eta\left(\sigma_{b, 2}\right) & =\{(\emptyset,\{P, Q, R\})\}, \\
\eta\left(\sigma_{b, 3}\right) & =\{(\{R\},\{P, Q\})\}, \\
\eta\left(\sigma_{c}\right) & =\{(\{R\},\{P, Q\})\},
\end{aligned}
$$

and

6. the labels of the positive and negative spiders are given by $\rho\left(\sigma_{a}\right)=a, \rho\left(\sigma_{b, 1}\right)=\rho\left(\sigma_{b, 2}\right)=\rho\left(\sigma_{b, 3}\right)=b$, and $\rho\left(\sigma_{c}\right)=c$.

We now proceed to define some useful syntactic notions. For example, in figure 3, the zone $(\{P, R\},\{Q\})$ is missing from $d_{6}$ (and $d_{5}$ ). Since $d_{6}$ is taken to assert that $R$ is disjoint from both $P$ and $Q$, the set $P \cap R \cap \bar{Q}$ represented by this zone must be empty.

Definition 2. Let $d=(L, Z, S h Z, E S, P S, N S, \eta, \rho)$ be an enhanced spider diagram. The missing zones of $d$ are elements of

$$
M Z(d)=\{(\text { in }, \text { out }) \in \mathcal{Z}: \text { in } \cup \text { out }=L\} \backslash Z .
$$

So, $d_{6}$ has three missing zones, giving $M Z\left(d_{6}\right)=\{(\{P, R\},\{Q\}),(\{Q, R\},\{P\}),(\{P, Q, R\}, \emptyset)\}$.

Missing zones need not be the only zones that represent empty sets. In particular, shading placed in zones enforces an upper bound on set cardinality: in a shaded region, all elements must be represented by existential or positive spiders. So, a shaded region containing no part of any such spider represents the empty set. There are no necessarily empty zones in $d_{6}$ but, in figure 4 , the diagram $d_{7}$ only contains empty zones (all zones are shaded and there are no existential or positive spiders).

Definition 3. Let $d=(L, Z, S h Z, E S, P S, N S, \eta, \rho)$ be an enhanced spider diagram. The empty zones of $d$ are elements of

$$
E Z(d)=\{z \in S h Z: \forall \sigma \in E S \cup P S \quad z \notin \eta(\sigma)\} .
$$

It is also useful to identify zones that represent sets in which an individual cannot lie, due to the information provided by negative spiders. For instance, in $d_{6}$ of figure 3 , $b$ is not in (the sets represented by) the three zones which contain $\bar{b}$. 
Definition 4. Let $d=(L, Z, S h Z, E S, P S, N S, \eta, \rho)$ be an enhanced spider diagram. Let $c$ be a constant from $\mathcal{C}$. The negative zones for $c$ in $d$ are elements of $N Z(c, d)$ where

$$
N Z(c, d)=\{z \in Z: \exists \sigma \in N S \quad \eta(\sigma)=\{z\} \wedge \rho(\sigma)=c\} .
$$

So, in $d_{6}$, we have:

- no negative zone for $a$ : $N Z\left(a, d_{6}\right)=\emptyset$,

- three negative zones for $b$ since there are three $\bar{b}$ s placed in single zones: $N Z\left(b, d_{6}\right)=$ $\{(\{Q\},\{P, R\}),(\emptyset,\{P, Q, R\}),(\{P\},\{P, Q\})\}$ and

- one negative zone for $c$, since there is one $\bar{c}$ placed in a single zone: $N Z\left(c, d_{6}\right)=\{(\{R\},\{P, Q\})\}$.
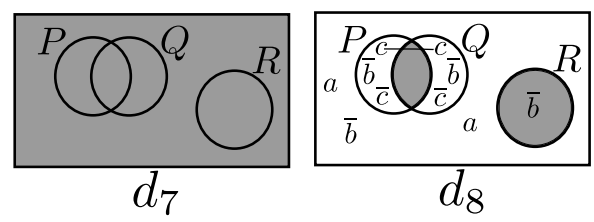

Figure 4. Inconsistency and clutter.

Our attention now turns to semantics. As is standard, we interpret the curve labels as subset of some (non-empty) universal set and constant symbols as elements.

Definition 5. An interpretation, $\mathcal{I}$, is a triple, $\mathcal{I}=$ $(U, \psi, \Psi)$, such that

1. $U$ is a non-empty set, called the universal set,

2. $\psi: \mathcal{C} \rightarrow U$ maps constants to elements in $U$, and

3. $\Psi: \mathcal{L} \rightarrow \mathbb{P} U$ maps curve labels to subsets of $U$.

The function $\Psi$ is extended to interpret zones and sets of zones (regions) as follows: for each zone, (in, out),

$$
\Psi(z)=\bigcap_{l \in \text { in }} \Psi(l) \cap \bigcap_{l \in \text { out }}(U \backslash \Psi(l))
$$

and for each region, $r, \Psi(r)=\bigcup_{z \in r} \Psi(z)$.

Given an interpretation, it can either agree with the intended intuitive meaning of a diagram or not. For instance, in figure 3, any interpretation where $\Psi(P) \cap \Psi(R) \neq \emptyset$ does not agree with the intended intuitive meaning of $d_{6}$ : because the curves $P$ and $R$ do not overlap, $d_{6}$ tells us that $\Psi(P) \cap \Psi(R)=\emptyset$. The semantics of diagrams are given by the set of interpretations that match our expectations of what the diagram expresses. We therefore give precise conditions under which an interpretation satisfies an enhanced spider diagram; satisfying interpretations are called models.
Definition 6. Let $d=(L, Z, S h Z, E S, P S, N S, \eta, \rho)$ be an enhanced spider diagram and let $\mathcal{I}=(U, \psi, \Psi)$ be an interpretation. Then $\mathcal{I}$ is a model for $d$ provided there exists a function, $\psi^{\prime}: E S \cup P S \cup N S \rightarrow U$, where, for each positive and negative spider, $\sigma$, in $P S \cup N S$ we have $\psi^{\prime}(\sigma)=\psi(\rho(c))$ and the following conditions hold.

1. Missing Zones Condition: missing zones represent empty sets, that is for all $z \in M Z(d), \Psi(z)=\emptyset$.

2. Shaded Zones Condition: shaded zones represent sets containing only elements represented by existential or positive spiders, that is for all $z \in S h Z$,

$$
\Psi(z) \subseteq\left\{\psi^{\prime}(\sigma): \sigma \in E S \cup P S\right\} .
$$

3. Spider Distinctness Condition no two existential or positive spiders represent the same element, that is for all $\sigma_{1}$ and $\sigma_{2}$ in $E S \cup P S$,

$$
\psi^{\prime}\left(\sigma_{1}\right)=\psi^{\prime}\left(\sigma_{2}\right) \Rightarrow \sigma_{1}=\sigma_{2} .
$$

4. Existential Spiders Condition each existential spider represents an element in the region in which it is placed, that is for all $\sigma$ in $E S, \psi^{\prime}(\sigma) \in \Psi(\eta(\sigma))$.

5. Positive Spiders Condition each positive spider represents an element in the region in which it is placed, that is for all $\sigma$ in $P S, \psi^{\prime}(\sigma) \in \Psi(\eta(\sigma))$.

6. Negative Spiders Condition each negative spider represents an element that is not some zone in the region in which it is placed, that is for all $\sigma$ in NS, there exists $z$ in $\eta(\sigma)$ where $\psi^{\prime}(\sigma) \notin \Psi(z)$.

Such a function $\psi^{\prime}$ that makes the above conditions true for $d$ is called valid. If $\mathcal{I}$ models $d$ then $\mathcal{I}$ satisfies $d$. Diagrams with no models are unsatisfiable.

The interpretation with $U=\{1,2,3,4\}, \psi(a)=1$, $\psi(b)=2, \psi(c)=3, \Psi(P)=\{1,2\}, \Psi(Q)=\{2,3\}$ and $\Psi(R)=\{4\}$ is a model for $d_{6}$. However, if instead $\Psi(Q)=\{1,2,3\}$ then the resulting interpretation fails to model $d_{6}$; for instance, $\psi(a)=1$ but, since the positive spider labelled $a$ is in the zone $(\{P\},\{Q, R\})$ and we have

$$
\begin{aligned}
\Psi(\{P\},\{Q, R\}) & =\Psi(P) \cap(U \backslash \Psi(Q)) \cap(U \backslash \Psi(R)) \\
& =\emptyset
\end{aligned}
$$

the positive spiders condition fails under any $\psi^{\prime}$ that agrees with $\psi$.

One important, yet straightforward to verify, insight is that distinct zones in a diagram represent disjoint sets. In essence, given two such zones $\left(i n_{1}\right.$, out $\left._{1}\right)$ and $\left(i n_{2}\right.$, out $\left._{2}\right)$, we know that $i n_{1} \cup o u t_{1}=i n_{2} \cup o u t_{2}$. Therefore, there is a label, $l$ say in $i n_{1} \cap$ out $_{2}$ or in $i n_{2} \cap o u t_{1}$. So one zone is a subset of $\Psi(l)$ and the other is a subset of $U \backslash \Psi(l)$. This leads us to lemma 1 : 
Lemma 1. Let $d=(L, Z, S h Z, E S, P S, N S, \eta, \rho)$ be an enhanced spider diagram. In all models, $I=(U, \psi, \Psi)$, for $d$,

$$
\Psi\left(z_{1}\right) \cap \Psi\left(z_{2}\right)
$$

for all zones $z_{1}$ and $z_{2}$ in $Z \cup M Z$. That is, distinct zones in $d$ or which are missing from $d$ represent disjoint sets.

We now present two results relating to, respectively, empty zones and negative zones. Firstly, we establish that empty zones represent empty sets in models:

Lemma 2. Let $d=(L, Z, S h Z, E S, P S, N S, \eta, \rho)$ be an enhanced spider diagram. In all models, $I=(U, \psi, \Psi)$, for $d, \Psi(E Z(d))=\emptyset$.

Proof. Suppose $I=(U, \psi, \Psi)$ is a model for $d$. Let $z \in$ $E Z(d)$. We show $\Psi(z)=\emptyset$. Since $I$ is a model for $d$, there exists a valid $\psi^{\prime}: E S \cup P S \cup N S \rightarrow U$ for $d$. Choose such a $\psi^{\prime}$. Since $z$ is in $E Z(d)$ we know that $z$ is shaded. So, by the shaded zones condition,

$$
\Psi(z) \subseteq\left\{\psi^{\prime}(\sigma): \sigma \in E S \cup P S\right\} .
$$

Let $\sigma$ be an existential or positive spider in $d$, so $\sigma \in E S \cup$ $P S$. Then, by the existential and positive spiders conditions for $d$ we know

$$
\psi^{\prime}(\sigma) \in \Psi(\eta(\sigma)) .
$$

Now, since $z$ is in $E Z(d)$, we know that $z \notin \eta(\sigma)$. Since distinct zones in $d$ represent disjoint sets (lemma 1), we deduce that

$$
\Psi(z) \cap \Psi(\eta(\sigma))=\emptyset .
$$

Therefore,

$$
\psi^{\prime}(\sigma) \notin \Psi(z) .
$$

Since $\sigma$ was an arbitrary existential or positive spider and the shaded zones condition holds (i.e. $\Psi(z)$ only contains elements represented by existential or positive spiders), we deduce that

$$
\Psi(z)=\emptyset .
$$

Hence $\Psi(E Z(d))=\emptyset$ as required.

Secondly, we show that, for any given spider label, $c$, its associated negative zones do not contain the individual represented by $c$. For instance, in $d_{6}$ we already saw that $N Z\left(b, d_{6}\right)$ includes $(\{Q\},\{P, R\})$. This zone is the location for a negative spider, $\sigma_{b, 1}$, labelled $b$, that is $\eta\left(\sigma_{b, 1}\right)=$ $\{(\{Q\},\{P, R\})\}$. In any model for $d_{6}$, the negative spiders condition tells us that $\psi^{\prime}(b)=\psi(b) \notin \Psi(\{Q\},\{P, R\})$. Importantly, the negative zones arise precisely from the negative spiders whose locations are single zones, from which the proof of the following lemma readily follows:

Lemma 3. Let $d=(L, Z, S h Z, E S, P S, N S, \eta, \rho)$ be an enhanced spider diagram. In all models, $I=(U, \psi, \Psi)$, for $d$, for all $c \in \mathcal{C}$, it is the case that $\psi(c) \notin \Psi(N Z(c, d))$.
Proof. Suppose $I=(U, \psi, \Psi)$ is a model for $d$ and let $c \in$ $\mathcal{C}$. If $N Z(c, d)=\emptyset$ then $\Psi(N Z(c, d))=\emptyset$ and we have $\psi(c) \notin \Psi(N Z(c, d))$. Alternatively, let $z \in N Z(c, d)$. We show $\psi(c) \notin \Psi(z)$. Since $I$ is a model for $d$, there exists a valid $\psi^{\prime}: E S \cup P S \cup N S \rightarrow U$ for $d$. Choose such a $\psi^{\prime}$. Now, since $z \in N Z(c, d)$, we know that there exists a negative spider, $\sigma$, in $d$ with label $c$ (i.e. $\rho(\sigma)=c$ ) and whose location is $z$ (i.e. $\eta(\sigma)=\{z\}$ ). By the negative spiders condition for $d$, we know that

$$
\psi^{\prime}(\sigma) \notin \Psi(z),
$$

as required. Hence $\psi(c) \notin \Psi(N Z(c, d))$.

Thus, the definitions of empty zones and negative zones have the expected properties in models. One further result will be useful to us, which establishes that in a model for diagram, $d$, each element in the universal set, $U$, must lie in some zone in $d$.

Lemma 4. Let $d=(L, Z, S h Z, E S, P S, N S, \eta, \rho)$ be an enhanced spider diagram. In all models, $I=(U, \psi, \Psi)$, for $d$,

$$
\bigcup_{z \in Z} \Psi(z)=\Psi(Z)=U
$$

\section{Inconsistency and Satisfiability}

A key motivation for this work is to explore the role of absence in clutter reduction. We begin by observing that every unsatisfiable diagram is semantically equivalent to a diagram containing no spiders. For example, in figure $4, d_{7}$ is inconsistent: every interpretation has a non-empty universal set yet, since $d_{7}$ is entirely shaded and contains no spiders, the shading and missing zones conditions can never both be satisfied (a non-empty universal set implies at least one zone represents a non-empty set). The diagram $d_{8}$ is also unsatisfiable, for any one of the following reasons:

1. There are two positive spiders both with the same label, $a$, meaning that the spider distinctness condition can never hold.

2. The negative $\bar{b}$ spiders together imply that $b$ must lie in $P \cap Q \cap \bar{R}$, yet this region is entirely shaded and contains no part of an existential or positive spider. Therefore, $d_{8}$ implies two contradictory statements: $b \in P \cap Q \cap \bar{R}$ and $P \cap Q \cap \bar{R}=\emptyset$.

3. The negative $\bar{c}$ spiders tell us that $c$ cannot lie in $P \cap \bar{Q} \cap$ $\bar{R}$ or in $Q \cap \bar{P} \cap \bar{R}$, yet the positive spider $c$ expresses $c \in(P \cap \bar{Q} \cap \bar{R}) \cup(Q \cap \bar{P} \cap \bar{R})$. Clearly both these assertions cannot be true at the same time.

Since $d_{8}$ is inconsistent, it is semantically equivalent to $d_{7}$, which is visually less cluttered. Since every inconsistent diagram is semantically equivalent to a diagram that is entirely shaded and contains no spiders, identifying necessary 
and sufficient conditions for unsatisfiability provides some insight into how negative spiders can lead to clutter reduction.

One important feature of the last example was that it was not possible to find zones that represent sets containing certain individuals. For instance, there was no zone for the individual $c$ since it was taken to be present in $(\{P\},\{Q, R\})$ or $(\{Q\},\{P, R\})$ yet absent from both $(\{P\},\{Q, R\})$ and $(\{Q\},\{P, R\})$. For a diagram to be satisfiable, for each constant, $c_{i}$, we must be able to select a zone that, in some model, represents a set containing the individual represented by $c_{i}$.

Definition 7. Let $d=(L, Z, S h Z, E S, P S, N S, \eta, \rho)$ be an enhanced spider diagram. A zone selection function for $d$ is a mapping, $f: E S \cup P S \cup N S \rightarrow Z$ which ensures the following hold:

1. the zone selected for each existential and positive spider is one of the zones in its location: for all $\sigma \in$ $E S \cup P S, f(\sigma) \in \eta(\sigma)$,

2. the zone selected for a negative spider cannot be a negative zone for its label: for all $\sigma \in N S, f(\sigma) \notin$ $N Z(\rho(\sigma), d)$ and

3. if a shaded zone is selected for a negative spider then it must also be selected for an existential or positive spider: for all $\sigma \in N S$, if $f(\sigma) \in S h Z$ then there exists $\sigma^{\prime} \in E S \cup P S$ such that $f\left(\sigma^{\prime}\right)=f(\sigma)$, and

4. spiders with the same label have the same zone selected: for all $\sigma_{1}, \sigma_{2}$ in $P S \cup N S$ if $\rho\left(\sigma_{1}\right)=\rho\left(\sigma_{2}\right)$ then $f\left(\sigma_{1}\right)=f\left(\sigma_{2}\right)$.

The zone selection function identifies, for each spider, a specific zone in the diagram, $d$. Any given zone selection function can be used to define a model for $d$, where the individuals represented by the spiders are in the sets represented by the selected zones. For the purposes of intuition, we consider each of the conditions of definition 7 . Condition 1 arises from the need for each existential and positive spider to represent an element in (the set represented by) one of the zones of its location. Condition 2 captures the fact that negative zones cannot contain, in a model for $d$, the individual represented by $\rho(\sigma)$. Condition 3 considers the interaction between negative spiders and shading. The zone selected for a negative spider, if shaded, cannot represent the empty set in a model. This is enforced by the requirement that some existential or positive spider has been assigned to that shaded zone. The last condition requires the same zone to be selected for spiders with a common label because such spiders represent the same individual.

Using $d_{6}$ in figure 3 as an example, adopting the previously given abstract syntax, we can define

$$
\begin{aligned}
& f\left(\sigma_{1}\right)=\{(\{P, Q\},\{R\})\}, \\
& f\left(\sigma_{a}\right)=\{(\{P\},\{Q, R\})\},
\end{aligned}
$$

$$
\begin{aligned}
f\left(\sigma_{b, 1}\right) & =\{(\{P, Q\},\{R\})\} \\
f\left(\sigma_{b, 2}\right) & =\{(\{P, Q\},\{R\})\} \\
f\left(\sigma_{b, 3}\right) & =\{(\{P, Q\},\{R\})\} \\
f\left(\sigma_{c}\right) & =\{(\{P, Q\},\{R\})\} .
\end{aligned}
$$

Under this zone selection function, a model can be generated for $d_{6}$ where $b$ and $c$ represent the same individual and that individual is in the set represented by the zone $\{(\{P, Q\},\{R\})\}$. Under any valid $\psi^{\prime}$ that respects $f$, this individual is also represented by $\sigma_{1}$, due to the presence of shading.

We are now in a position to define the notion of (in)consistency:

Definition 8. Let $d=(L, Z, S h Z, E S, P S, N S, \eta, \rho)$ be an enhanced spider diagram. Whenever the following conditions all hold $d$ is consistent:

1. If all of the zones in $Z$ are shaded then there is at least one existential or positive spider.

2. No two positive spiders have the same label, that is, the function $\rho$ is injective when its domain is restricted to $P S$.

3. There exists a zone selection function, $f$, for $d$.

\section{If $d$ is not consistent then $d$ is inconsistent.}

So, $d_{6}$ in figure 3 is consistent whereas $d_{7}$ and $d_{8}$ in figure 4 are inconsistent.

In order to prove that a consistent diagram, $d$ is satisfiable, our approach is to construct an interpretation that is a model for $d$. To do so, we make use of a foot selection function, which we know exists since $d$ is consistent. The first part of this task is to construct a suitable universal set. Now, for each existential spider and positive spider in $d$, we require the existence of a distinct element in $U$. So, our strategy is to include all of the existential spiders and the positive spiders in $U$. However, this alone does not ensure enough elements will be in $U$ for all of the spiders. In particular, negative spiders may require the existence of elements in non-shaded zones where there is no element arising from an existential or positive spider. Thus, our universal set will also include, as elements, all of the non-shaded zones. In fact, we will establish that this provides sufficient elements in $U$.

Once $U$ is constructed, we must build a suitable mapping from constant symbols to elements in $U$. For the constants that are used to label positive spiders this is straightforward: we map the label to the (unique) positive spider in $d$ (and, thus, in $U$ ) with that label. Things are less straightforward for a constant, $c$, that does not label a positive spider but does appear on one or more negative spiders. As indicated above, if the selected zone for such spiders is non-shaded then we simply map the associated constant symbol to that 
zone. What, then, if the selected zone is shaded? Here, we focus on the third requirement of a foot selection function: if a shaded zone is selected for a negative spider then it must also be selected for a positive or existential spider. This requirement arises since, in shaded zones, all elements must be represented by existential or positive spiders. So, to map a constant symbol, $c$, appearing in $d$ on negative spiders only, whose selected zone is shaded, we must choose one of these elements of $U$ (i.e an existential or positive spider with the same selected zone) to be the interpretation of $c$. Our next definition allows us to pair such negative spiders with existential or positive spiders:

Definition 9. Let $d=(L, Z, S h Z, E S, P S, N S, \eta, \rho)$ be a consistent enhanced spider diagram with foot selection function $f$. A selected negative spider mapping for $d$ given $f$ is a function, $s: N S^{\prime} \rightarrow E S \cup P S$ which ensures

$$
s(\sigma)=\sigma^{\prime} \Rightarrow f(\sigma)=f\left(\sigma^{\prime}\right)
$$

where

$$
N S^{\prime}=\left\{\sigma \in N S: \neg \exists \sigma^{\prime} \in P S \rho(\sigma)=\rho\left(\sigma^{\prime}\right) \wedge f(\sigma) \in S h Z\right\} .
$$

Lemma 5. Let $d=(L, Z, S h Z, E S, P S, N S, \eta, \rho)$ be a consistent enhanced spider diagram with foot selection function $f$. Then $d$, given $f$, has a selected negative spider mapping.

Proof Sketch. The proof follows trivially from condition 3 of definition 7.

Definition 10. Let $d=(L, Z, S h Z, E S, P S, N S, \eta, \rho)$ be a consistent enhanced spider diagram with a zone selection function, $f$, and selected negative spider mapping, $s . A$ standard interpretation, $I=(U, \psi, \Psi)$, for d given $f$ and $s$ is defined as follows.

1. the universal set comprises the non-shaded zones, the existential spiders and the positive spiders:

$$
U=(Z \backslash S h Z) \cup E S \cup P S
$$

2. $\psi: \mathcal{C} \rightarrow U$ is defined as follows, where $u$ is an arbitrary element in $U$ :

$$
\psi(c)= \begin{cases}\sigma & \text { when } \sigma \text { is in PS with } \rho(\sigma)=c \\ f(\sigma) & \sigma \text { is in } N S, \text { no positive spider has } \\ & \text { label } c, \rho(\sigma)=c \text { and } f(\sigma) \in Z \backslash S h Z \\ s(\sigma) & \sigma \text { is in } N S^{\prime} \text { with } \rho(\sigma)=c \\ u & \text { otherwise. }\end{cases}
$$

3. $\Psi: \mathcal{L} \rightarrow \mathbb{P} U$ is defined as follows:

$$
\begin{aligned}
\Psi(l)= & \{\sigma \in U \backslash Z: f(\sigma)=(\text { in }, \text { out }) \wedge l \in \text { in }\} \cup \\
& \{(\text { in }, \text { out }) \in U \cap Z: l \in \text { in }\} .
\end{aligned}
$$

Lemma 6. Let $d=(L, Z, S h Z, E S, P S, N S, \eta, \rho)$ be a consistent enhanced spider diagram with a zone selection function, $f$, and selected negative spider mapping, s. Let $I=(U, \psi, \Psi)$ be a standard interpretation for d given $f$ and s. Let $z \in Z \cup M Z$. Then

$$
\Psi(z)=\{\sigma \in U \backslash Z: f(\sigma)=z\} \cup(U \cap\{z\}) .
$$

Theorem 1. Let $d=(L, Z, S h Z, E S, P S, N S, \eta, \rho)$ be a consistent enhanced spider diagram with a zone selection function, $f$, and selected negative spider mapping, $s$. Let $I=(U, \psi, \Psi)$ be a standard interpretation for $d$ given $f$ and s. Then I is a model for $d$.

Proof. We start by showing that $U$ is not empty, since this is a requirement for $I$ to be an interpretation. Trivially, if there is a non-shaded zone then $U$ is non-empty. Else, all zones are shaded. Since $d$ is consistent, this implies that there is at least one existential or positive spider in $d$. By construction, such a spider is in $U$. In both cases, $U$ is nonempty as required.

We now show that the conditions for $U$ to be a model for $d$ are met. We start by defining a function

$$
\psi^{\prime}: E S \cup P S \cup N S \rightarrow U
$$

by

$$
\psi^{\prime}(\sigma)= \begin{cases}\sigma & \begin{array}{l}
\text { if } \sigma \in E S \cup P S \\
\sigma^{\prime}
\end{array} \\
\text { if } \sigma \in N S \text { and } \\
\text { there is a } \sigma^{\prime} \text { in } P S \text { where } \rho(\sigma)=\rho\left(\sigma^{\prime}\right) \\
f(\sigma) \begin{array}{l}
\sigma \text { is in } N S, \text { no positive spider has } \\
\text { label } \rho(\sigma), \text { and } f(\sigma) \in Z \backslash S h Z \\
s(\sigma)
\end{array} \quad \begin{array}{l}
\sigma \text { is in } N S^{\prime} .
\end{array}\end{cases}
$$

Next, we show that $\psi^{\prime}$ is valid. Firstly, consider the missing zones condition. Let $z$ be a missing zone. By lemma 6 , we know that

$$
\Psi(z)=\{\sigma \in U \backslash Z: f(\sigma)=z\} \cup(U \cap\{z\})
$$

Since $z$ is missing, $z$ is not in $U$, so

$$
\Psi(z)=\{\sigma \in U \backslash Z: f(\sigma)=z\} .
$$

But the zone selection function, $f$, has codomain $Z$, so no spider maps to $z$ under $f$. Therefore $\Psi(z)=\emptyset$ as required. Hence the missing zones condition holds.

Next we consider the shaded zones condition. Let $z$ be a shaded zone. By lemma 6, we know that

$$
\Psi(z)=\{\sigma \in U \backslash Z: f(\sigma)=z\} \cup(U \cap\{z\})
$$

Since $z$ is shaded, $z$ is not in $U$, so

$$
\Psi(z)=\{\sigma \in U \backslash Z: f(\sigma)=z\} .
$$


From this it follows that

$$
\Psi(z) \subseteq E S \cup P S
$$

and, noting that $\psi^{\prime}$ maps existential and positive spiders to themselves (i.e. over the restricted domain $E S \cup P S, \psi^{\prime}$ is the identity function) we have

$$
\Psi(z) \subseteq\left\{\psi^{\prime}(\sigma): \sigma \in E S \cup P S\right\}
$$

as required. Hence the shaded zones condition holds.

For the spider distinctness condition, we note that $\psi^{\prime}$ is injective when its domain is restricted to $E S \cup P S$. Hence the spider distinctness condition holds. There are three remaining conditions to verify, all related to spiders. Let $\sigma$ be an existential spider. Consider $\Psi(f(\sigma))$. We know

$$
\Psi(f(\sigma))=\left\{\sigma^{\prime} \in U \backslash Z: f\left(\sigma^{\prime}\right)=f(\sigma)\right\} \cup(U \cap\{f(\sigma)\})
$$

by lemma 6. Clearly, $\sigma$ is in $\Psi(f(\sigma))$ and, since $\sigma=\psi^{\prime}(\sigma)$, it follows that

$$
\psi^{\prime}(\sigma) \in \Psi(f(\sigma)) .
$$

Moreover, since $f$ is a zone selection function, $f(\sigma)$ is in $\eta(\sigma)$. We deduce that

$$
\psi^{\prime}(\sigma) \in \Psi(f(\sigma)) \subseteq \Psi(\eta(\sigma)) .
$$

Therefore the existential spiders condition holds. The positive spiders condition similarly holds.

All that remains is to show that the negative spiders condition holds. Let $\sigma$ be a negative spider. If $\eta(\sigma)$ contains at least two zones then the negative spiders condition trivially holds: $\psi^{\prime}(\sigma)$ can be in at most one of the sets represented by the two or more zones. We can assume, then, in what follows that $|\eta(\sigma)|=1$. There are three cases to consider. In all cases, we aim to show that $\psi^{\prime}(\sigma) \notin \Psi(z)$ where $\eta(\sigma)=\{z\}$.

Case (1): $\sigma \in N S$ and there is a $\sigma^{\prime}$ in $P S$ where $\rho(\sigma)=\rho\left(\sigma^{\prime}\right)$. Here, $\psi^{\prime}(\sigma)=\psi^{\prime}\left(\sigma^{\prime}\right)$ by definition and, by the reasoning above we know that

$$
\psi^{\prime}(\sigma)=\psi^{\prime}\left(\sigma^{\prime}\right) \in \Psi\left(f\left(\sigma^{\prime}\right)\right) .
$$

Moreover, since $f$ is a foot selection function and $\rho(\sigma)=\rho\left(\sigma^{\prime}\right)$ we also know that $f(\sigma)=f\left(\sigma^{\prime}\right)$. Thus,

$$
\psi^{\prime}(\sigma)=\psi^{\prime}\left(\sigma^{\prime}\right) \in \Psi\left(f\left(\sigma^{\prime}\right)\right)=\Psi(f(\sigma)) .
$$

Since $\eta(\sigma)$ contains a single zone, $z$, by assumption, it follows that $z \in N Z(\rho(\sigma), d)$. Again, since $f$ is a zone selection function, we see that

$$
f(\sigma) \notin N Z(\rho(\sigma), d)
$$

and we can deduce that $f(\sigma) \neq z$. Hence $f(\sigma) \notin$ $\eta(\sigma)$, so $\psi^{\prime}(\sigma) \notin \Psi(\eta(\sigma))$ as required.
Case (2): $\sigma$ is in $N S$, no positive spider has label $\rho(\sigma)$, and $f(\sigma) \in Z \backslash S h Z$. In this case, $\psi^{\prime}(\sigma)=f(\sigma)$, by definition. We know, by lemma 6 , that

$$
\Psi(f(\sigma))=\left\{\sigma^{\prime} \in U \backslash Z: f\left(\sigma^{\prime}\right)=f(\sigma)\right\} \cup(U \cap\{f(\sigma)\}) .
$$

Since $U$ includes all non-shaded zones, and $f(\sigma)$ is such a zone, we see that

$$
\psi^{\prime}(\sigma)=f(\sigma) \in \Psi(f(\sigma))
$$

As $f$ is a foot selection function, $f(\sigma)$ is not in $N Z(\rho(\sigma), d))$. Moreover, because $\eta(\sigma)$ contains only one zone, $z$, (by assumption), we know that $z$ is in $N Z(\rho(\sigma), d))$. Now, since distinct zones in $d$ represent disjoint sets, we have

$$
\psi^{\prime}(\sigma) \notin \Psi(z)=\Psi(\eta(\sigma))
$$

as required.

Case (3): $\sigma$ is in $N S^{\prime}$. Here, we use the fact that $s(\sigma)=$ $\sigma^{\prime}$, for some $\sigma^{\prime}$ in $E S \cup P S$, where $f(\sigma)=f\left(\sigma^{\prime}\right)$. We already saw that

$$
\psi^{\prime}\left(\sigma^{\prime}\right) \in \Psi\left(f\left(\sigma^{\prime}\right)\right)=\Psi(f(\sigma)) .
$$

Now, $\psi^{\prime}\left(\sigma^{\prime}\right)=\sigma^{\prime}$, by construction, and $\psi^{\prime}(\sigma)=\sigma^{\prime}$, so

$$
\psi^{\prime}(\sigma) \in \Psi(f(\sigma)) .
$$

Again, as the location, $\eta(\sigma)$, contains a single zone and $f(\sigma)$ is not a negative zone for $\rho(\sigma)$ in $d$, it follows that

$$
\psi^{\prime}(\sigma) \notin \Psi(\eta(\sigma)) .
$$

Hence, in all cases the negative spiders condition holds and we have shown that $I$ is a model for $d$.

We can now state and prove the main result of this section:

Theorem 2. Let $d=(L, Z, S h Z, E S, P S, N S, \eta, \rho)$ be an enhanced spider diagram. Then $d$ is consistent if and only if $d$ is satisfiable.

Proof. For the first part of the proof, assume $d$ is consistent. Then, by theorem 1, a standard model satisfies $d$. Hence, $d$ is satisfiable.

For the converse, assume that $d$ is satisfiable. We must show that $d$ is consistent. Let $I=(U, \psi, \Psi)$ be a model for $d$ and assume $\psi^{\prime}: E S \cup P S \cup N S \rightarrow U$ is a valid mapping of spiders to universal set elements. We now consider three cases, relating to the conditions for $d$ to be consistent.

Firstly, then, assume that $d$ is entirely shaded. Our task is to show that $d$ contains an existential or positive spider. Since $I$ is an interpretation, $U$ is not empty. Let $e$ be an 
element of $U$. Then there is some zone, $z$, in $d$ where $e \in$ $\Psi(z)$, by lemma 4 . By assumption, $z$ is shaded so

$$
\Psi(z) \subseteq\{\psi(\sigma): \sigma \in E S \cup P S\}
$$

by the shaded zones condition. Therefore

$$
e \in\{\psi(\sigma): \sigma \in E S \cup P S\}
$$

from which it trivially follows that there is a spider in $E S$ or $P S$ as required.

Secondly, we must show that no two positive spiders have the same label. Suppose there are two positive spiders, $\sigma_{1}$ and $\sigma_{2}$, with the same label. By the spider distinctness condition, we know that

$$
\psi^{\prime}\left(\sigma_{1}\right) \neq \psi^{\prime}\left(\sigma_{2}\right)
$$

But since $\psi^{\prime}$ is valid, we also know that

$$
\psi^{\prime}\left(\sigma_{1}\right)=\psi\left(\rho\left(\sigma_{1}\right)\right)=\psi\left(\rho\left(\sigma_{2}\right)\right)=\psi^{\prime}\left(\sigma_{2}\right)
$$

which is a contradiction. Hence no two positive spiders have the same label.

Lastly, we must show that there is a zone selection function for $d$. We construct $f: E S \cup P S \cup N S \rightarrow Z$ and show that $f$ is such a function. Consider, for each spider, $\sigma$, in $E S \cup P S \cup N S, \psi^{\prime}(\sigma)$. Since $\psi^{\prime}(\sigma)$ is in $U$, we know, by lemma 4 and since distinct zones in $d$ represent disjoint sets, there is a unique zone, $z$, such that $\psi^{\prime}(\sigma) \in \Psi(z)$. We define, for each $\sigma$ in $E S \cup P S \cup N S$,

$$
f(\sigma)=z
$$

where $\psi^{\prime}(\sigma) \in \Psi(z)$ and $z \in Z$. We must now verify that $f$ satisfies the four conditions required of a zone selection function.

1. We must show that the zone selected for each existential and positive spider, $\sigma$, is a zone in its location, that is $f(\sigma) \in \eta(\sigma)$. By definition, $f(\sigma)$ is the zone whose interpretation under $\Psi$ contains $\psi^{\prime}(\sigma)$. By the existential and positive spiders conditions, we know that $\psi^{\prime}(\sigma) \in \Psi(\eta(\sigma))$. So, we have both

$$
\begin{aligned}
& \psi^{\prime}(\sigma) \in \Psi(f(\sigma)) \quad \text { and } \\
& \psi^{\prime}(\sigma) \in \Psi(\eta(\sigma))
\end{aligned}
$$

Since distinct zones in $d$ represent disjoint sets, it follows that $f(\sigma) \in \eta(\sigma)$ as required.

2. Now we must show that the zone, $f(\sigma)$, selected for a negative spider, $\sigma$ is not in $N Z(\rho(\sigma), d)$. Let $\sigma_{1}, \ldots, \sigma_{n}$ be all of the negative spiders in $d$ with the same label as $\sigma$. Then we know that $\psi^{\prime}\left(\sigma_{1}\right)=\ldots=\psi^{\prime}\left(\sigma_{n}\right)$, so all of these spiders have the same zone, $z$, selected under $f$. For each spider, either $z$ is not in it's location or, by the negative spiders condition, there is another zone in $\eta(z)$. For $z$ to be a negative zone for $\sigma$, it would have to be the case that $z$ is the only zone in the location of one $\sigma_{1}, \ldots$, or $\sigma_{n}$. As we have just seen, this is not the case, so $z$ is not negative for $\sigma$. That is $f(\sigma) \notin N Z(\rho(\sigma), d)$, as required.

3. Suppose that a shaded zone is selected by $f$ for some negative spider, $\sigma$. We must show that $f(\sigma)$ is also selected for some existential or positive spider. By the shaded zones condition,

$$
\Psi(f(\sigma)) \subseteq\left\{\psi^{\prime}\left(\sigma^{\prime}\right): \sigma^{\prime} \in E S \cup P S\right\} .
$$

Moreover, by the definition of $f, \psi^{\prime}(\sigma) \in \Psi(f(\sigma))$, so

$$
\psi^{\prime}(\sigma) \in\left\{\psi^{\prime}\left(\sigma^{\prime}\right): \sigma^{\prime} \in E S \cup P S\right\} .
$$

Choose $\sigma^{\prime}$ in $E S \cup P S$ such that $\psi^{\prime}(\sigma)=\psi^{\prime}\left(\sigma^{\prime}\right)$. Then $\psi^{\prime}\left(\sigma^{\prime}\right) \in \Psi(f(\sigma))$ and, since distinct zones in $d$ represent disjoint sets, we deduce that $f\left(\sigma^{\prime}\right)=f(\sigma)$, as required.

4. Lastly, we show that if two positive or negative spiders, $\sigma_{1}$ and $\sigma_{2}$, have the same label then they have the same zone selected. Trivially,

$$
\psi^{\prime}\left(\sigma_{1}\right)=\psi^{\prime}\left(\sigma_{2}\right)
$$

in other words $\sigma_{1}$ and $\sigma_{2}$ represent the same element. Therefore, both $\sigma_{1}$ and $\sigma_{2}$ represent an element in the set denoted by some zone $z$. Thus $f\left(\sigma_{1}\right)=f\left(\sigma_{2}\right)=z$ as required.

Hence all four conditions are met by $f$ and we deduce that $f$ is a zone selection function. Therefore $d$ is consistent. Thus, $d$ is consistent if and only if $d$ is satisfiable.

To conclude this section, we add an inference rule for inconsistency:

Inference Rule 1 (Inconsistency). Let $d=$ $(L, Z, S h Z, E S, P S, N S, \eta, \rho)$ be an inconsistent enhanced spider diagram. Let $d^{\prime}$ be any enhanced spider diagram. Then d may be replaced by $d^{\prime}$.

\section{Inference Rules for Spiders}

The goal of this section is to introduce inference rules that can later be used to reduce clutter in enhanced spider diagrams. These inference rules focus on spiders only. It is therefore helpful to introduce transformations on diagrams that remove and add spiders. In what follows we use $\mid$ to indicate a domain restriction. 
Transformation 1. Let $d=(L, Z, S h Z, E S, P S, N S, \eta, \rho)$ be an enhanced spider diagram. Let $\sigma$ be a spider in $S(d)$. We define a spider removal operation on $d$ :

$$
\begin{aligned}
& d-\sigma=\left(L, Z, S h Z, E S \backslash\{\sigma\}, P S \backslash\{\sigma\}, N S \backslash\{\sigma\}, \eta^{\prime}, \rho^{\prime}\right) \\
& \text { where } \eta^{\prime}=\left.\eta\right|_{S \backslash\{\sigma\}} \text {, and } \rho^{\prime}=\left.\rho\right|_{(P S \cup N S) \backslash\{\sigma\}} .
\end{aligned}
$$

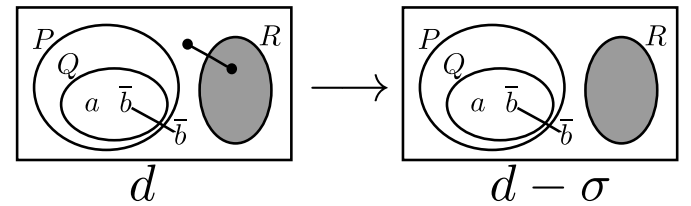

Figure 5. Removing a spider.

For example, in figure 5, the existential spider, $\sigma$, is removed from $d$ to give $d-\sigma$. This removal transformation only needs to 'know' which spider to remove. However, when adding a spider, we need to know the location in which it is to be placed and, if it is positive or negative, its label must be supplied. Figure 6 shows three applications of the spider addition transformation. In each case, a spider is added to the region $r=\{(\{P\},\{Q, R\}),(\emptyset,\{P, Q, R\})\}$. In the first case, the existential spider $\sigma_{e}$ is added. In the second and third cases, a positive and, respectively, negative spider ( $\sigma_{a}$ and $\sigma_{\bar{a}}$ resp.) is added with the label $a$.

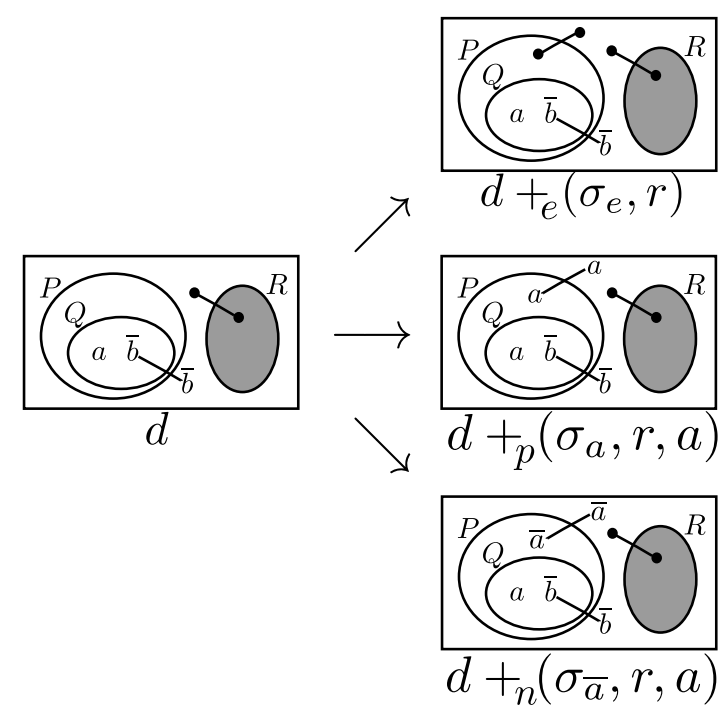

Figure 6. Adding spiders.

Transformation 2. Let $d=(L, Z, S h Z, E S, P S, N S, \eta, \rho)$ be an enhanced spider diagram. Let $\sigma$ be an element that is not in $S(d)$ (i.e. a fresh spider), let $c$ be a constant in $\mathcal{C}$, and let $r$ be a subset of $Z$. We define three spider addition operations on $d$ :
1. $d+_{e}(\sigma, r)=(L, Z, S h Z, E S \cup\{\sigma\}, P S, N S, \eta \cup$ $\{(\sigma, r)\}, \rho)$

2. $d+_{p}(\sigma, r, c)=(L, Z, S h Z, E S, P S \cup\{\sigma\}, N S, \eta \cup$ $\{(\sigma, r)\}, \rho \cup\{(\sigma, c)\})$

3. $d+{ }_{n}(\sigma, r, c)=(L, Z, S h Z, E S, P S, N S \cup\{\sigma\}, \eta \cup$ $\{(\sigma, r)\}, \rho \cup\{(\sigma, c)\})$.

These transformations will now be used to define inference rules that delete spiders, shrink spiders, and swap spiders; we do not provide rules for adding spiders since such a transformation increases visual clutter and our focus is on reducing clutter. Importantly, all these rules are equivalences: the diagram to which the rule is applied has the same models as the resulting diagram. The fact that the rules are equivalences means we can explore different representations of information using different types of spider. In addition, the rules are only defined for consistent diagrams; when applied to inconsistent diagrams they need not be equivalences. In section 6 we will discuss the impact of negative spiders on clutter reduction.

Firstly we introduce three inference rules that allow spiders to be deleted. Clearly, deleting spiders allows clutter to be reduced. We start by observing that, in any diagram $d$, and for constant, $c$, in $\mathcal{C}$, the individual represented by $c$ lies in the set represented by $Z \backslash E Z$. Moreover, the individual cannot lie in the set represented by $N Z(c, d)$, so we can make the stronger assertion that the individual lies in the set represented by $(Z \backslash E Z) \backslash N Z(c, d)$. We use this insight in the first rule, which focuses on existential spiders and exploits absence information.

For example, in figure 7, the existential spider, $\sigma$, in the zone $(\emptyset,\{P, Q\})$ can be deleted. It is the only spider in this non-shaded location and, moreover, the negative zones for $c$ are precisely all of the zones except $(\emptyset,\{P, Q\})$. Therefore, on deleting $\sigma$ the information that $(\emptyset,\{P, Q\})$ does not represent the empty set, which is directly asserted by $\sigma$, can be deduced from the three negative spiders labelled $c$. Neither of the other two existential spiders can be deleted. Deleting the existential spider in the shaded zone $(\{P\},\{Q\})$ would not be sound: in models for $d$, this zone contains exactly two elements, deleting this spider results in a diagram in which all models require this zone to contain just one element. The other existential spider, which is placed in two zones, tells us that there is some element in the respective set that is different from the individual $b$. Thus, deleting this other existential spider, whilst sound, weakens information and does not result in a semantically equivalent diagram.

Inference Rule 2 (Delete Existential Spider). Let $d=$ ( $L, Z, S h Z, E S, P S, N S, \eta, \rho)$ be a consistent enhanced spider diagram. Let $\sigma$ be an existential spider in $d$. If

1. there are no other existential or positive spiders in $d$, whose location overlaps with $\sigma$ 's, that is for all $\sigma^{\prime}$ in $(E S \cup P S) \backslash\{\sigma\}, \eta(\sigma) \cap \eta\left(\sigma^{\prime}\right)=\emptyset$, 


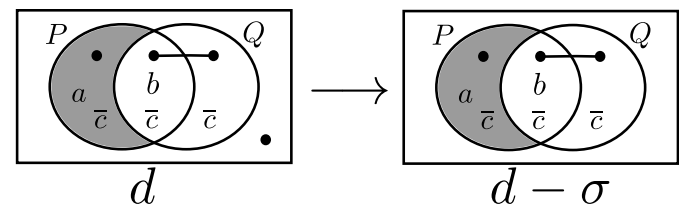

Figure 7. Delete existential spider.

2. $\sigma$ 's location does not include shaded zones, that is

$$
\eta(\sigma) \cap S h Z=\emptyset,
$$

and

3. some constant, $c$, must represent an individual in the set denoted by $\eta(\sigma)$, that is

$$
(Z \backslash E Z) \backslash N Z(c, d) \subseteq \eta(\sigma) .
$$

then $d$ may be replaced by $d-\sigma$.

Lemma 7. Delete existential spider is sound and results in a semantically equivalent diagram.

Proof. Suppose we delete existential spider $\sigma$ from $d$ to give $d-\sigma$. It is trivial to show that $d$ semantically entails $d-\sigma$, in part since the location of $\sigma$ does not include any shaded zones. Therefore, we focus on the converse: $d-\sigma$ semantically entails $d$. Let $I=(U, \psi, \Psi)$ be a model for $d-\sigma$ with valid $\psi^{\prime}:(E S \backslash\{\sigma\}) \cup P S \cup N S \rightarrow U$. We show that $I$ is a model for $d$. Our strategy is as follows:

1. show $\Psi(\eta(\sigma)) \neq \emptyset$,

2. choose $e \in \Psi(\eta(\sigma))$ and extend $\psi^{\prime}$ to $\psi^{\prime \prime}$ where $\psi^{\prime \prime}(\sigma)=e$, and

3. show $\psi^{\prime \prime}$ is valid for $d$.

Firstly, then, we show show $\Psi(\eta(\sigma)) \neq \emptyset$. Consider the constant, $c$, that ensures

$$
(Z \backslash E Z) \backslash N Z(c, d) \subseteq \eta(\sigma) .
$$

We know that $\psi(c)$ is an element of $U$ and, by lemma 4, $\psi(c) \in \Psi(z)$ for some $z \in Z$. Moreover, by lemma $2, z$ is not in $E Z(d)$. Thus far, we know that $z$ is in $Z \backslash E Z$. By lemma 3 we further know that $\psi(c)$ is not in $\Psi(N Z(c, d))$. Since distinct zones represent disjoint sets, we deduce that $z$ is not in $N Z(c, d)$. Hence,

$$
z \in(Z \backslash E Z) \backslash N Z(c, d) \subseteq \eta(\sigma)
$$

so

$$
\psi(c) \in \Psi(z) \subseteq \Psi(\eta(\sigma)) .
$$

It follows that $\Psi(\eta(\sigma)) \neq \emptyset$. by

Choose $e \in \Psi(\eta(\sigma))$ and define $\psi^{\prime \prime}: E S \cup P S \cup N S \rightarrow U$

$$
\psi^{\prime \prime}\left(\sigma^{\prime}\right)= \begin{cases}\psi^{\prime}\left(\sigma^{\prime}\right) & \text { if } \sigma^{\prime} \neq \sigma \\ e & \text { otherwise. }\end{cases}
$$

We show $\psi^{\prime \prime}$ is valid for $d$, but first we consider the missing zones condition. Trivially, this holds for $d$ in $I$ as it is identical for $d$ and $d-\sigma$. Consider now the shaded zones condition. Let $z$ be a shaded zone in $d$. Then $z$ is shaded in $d-\sigma$. By the shaded zones condition for $d-\sigma$ we know

$$
\Psi(z) \subseteq\left\{\psi^{\prime}\left(\sigma^{\prime}\right): \sigma^{\prime} \in(E S \backslash\{\sigma\}) \cup P S\right\} .
$$

Therefore

$$
\Psi(z) \subseteq\left\{\psi^{\prime \prime}\left(\sigma^{\prime}\right): \sigma^{\prime} \in E S \cup P S\right\}
$$

as required. Hence the shaded zones condition holds for $d$.

For the spider distinctness condition, let $\sigma_{1}$ and $\sigma_{2}$ be distinct existential or positive spiders in $d$. If both of them are in $d-\sigma$ then we know $\psi^{\prime}\left(\sigma_{1}\right) \neq \psi^{\prime}\left(\sigma_{2}\right)$ implying that $\psi^{\prime \prime}\left(\sigma_{1}\right) \neq \psi^{\prime \prime}\left(\sigma_{2}\right)$. Assume without loss of generality, that $\sigma_{2}=\sigma$. We must show that $\psi^{\prime \prime}\left(\sigma_{1}\right) \neq \psi^{\prime \prime}(\sigma)$. We know already that $\psi^{\prime \prime}(\sigma)$ is in $\Psi(\eta(\sigma))$. Since $\psi^{\prime}$ is valid for $d-\sigma$, we also know that

$$
\psi^{\prime}\left(\sigma_{1}\right)=\psi^{\prime \prime}\left(\sigma_{1}\right) \in \Psi\left(\eta\left(\sigma_{1}\right)\right) .
$$

In $d$, by the definition of the delete existential spider inference rule, the spider $\sigma$ has a location that is disjoint from all other spider locations. Therefore

$$
\eta\left(\sigma_{1}\right) \cap \eta(\sigma)=\emptyset .
$$

Thus

$$
\psi^{\prime \prime}\left(\sigma_{1}\right) \notin \Psi(\eta(\sigma))
$$

and we also have

$$
\psi^{\prime \prime}(\sigma)=e \in \Psi(\eta(\sigma)) .
$$

It follows that $\psi^{\prime \prime}\left(\sigma_{1}\right) \neq \psi^{\prime \prime}(\sigma)$, as required. Hence the spider distinctness condition holds for $d$.

Trivially, the existential spiders condition holds for $d$, noting that it is identical to that for $d-\sigma$ except for the extra spider $\sigma$ (and we know, by the construction of $\psi^{\prime \prime}$ that $\left.\psi^{\prime \prime}(\sigma) \in \Psi(\eta(\sigma))\right)$. Similarly, the positive and negative spiders conditions hold for $d$, as they are identical conditions in $d$ and $d-\sigma$. Hence $\psi^{\prime \prime}$ is valid for $d$ and it follows that $I$ models $d$. Therefore, delete existential spider is sound and $d-\sigma$ is semantically equivalent to $d$.

Intuitively, the delete existential spider rule can be applied when we know the element represented by $c$ is in the set represented by $\eta(\sigma)$. However, it is important that no other existential or positive spiders have a location that 
overlaps with $\sigma$, essentially because negative spiders provide no distinctness information. The next rule, which allows the deletion of a positive spider, is similar: a positive spider can be deleted when the information it provides is also given by negative spiders:

Inference Rule 3 (Delete Positive Spider). Let $d=$ $(L, Z, S h Z, E S, P S, N S, \eta, \rho)$ be a consistent enhanced spider diagram. Let $\sigma$ be a positive spider in $d$. If

1. there are no other existential or positive spiders in $d$, whose location overlaps with $\sigma$ 's, that is for all $\sigma^{\prime}$ in $(E S \cup P S) \backslash\{\sigma\}, \eta(\sigma) \cap \eta\left(\sigma^{\prime}\right)=\emptyset$,

2. the only shaded zones in $\sigma$ 's location are in $N Z(\rho(\sigma), d)$, that is

$$
\eta(\sigma) \cap S h Z \subseteq N Z(\rho(\sigma), d),
$$

and

3. the negative zones for $\rho(\sigma)$ indicate that $\sigma$ must represent an individual in the set denoted by $\eta(\sigma)$ :

$$
(Z \backslash E Z) \backslash N Z(\rho(\sigma), d) \subseteq \eta(\sigma) .
$$

then $d$ may be replaced by $d-\sigma$.

Lemma 8. Delete positive spider is sound and results in a semantically equivalent diagram.

Proof Sketch. The proof is similar to that for the delete existential spider inference rule, so we just provide a sketch to illustrate key differences. Suppose we delete positive spider $\sigma$ from $d$ to give $d-\sigma$. It is trivial to show that $d$ semantically entails $d-\sigma$, in part since the location of $\sigma$ does not include any shaded zones other than those which are negative for $\rho(\sigma)$. Therefore, we focus on the converse: $d-\sigma$ semantically entails $d$. Let $I=(U, \psi, \Psi)$ be a model for $d-\sigma$ with valid $\psi^{\prime}: E S \cup(P S \backslash\{\sigma\}) \cup N S \rightarrow U$. We need to show that $I$ is a model for $d$. The strategy is as follows:

1. $\operatorname{show} \Psi(\eta(\sigma)) \neq \emptyset$,

2. show $\psi(\rho(\sigma)) \in \Psi(\eta(\sigma))$ and extend $\psi^{\prime}$ to $\psi^{\prime \prime}$ where $\psi^{\prime \prime}(\sigma)=\psi(\rho(\sigma)$, and

3. show $\psi^{\prime \prime}$ is valid for $d$.

Noting the similarity to lemma 7 , the rest of the details are straightforward.

Interestingly, negative spiders can always be deleted when they have multi-zone locations: if a negative spider, $\sigma$, has location $\left\{z_{1}, z_{2}\right\}$ for example, then this spider expresses that $\psi(\rho(\sigma)) \notin \Psi\left(z_{1}\right)$ or $\psi(\rho(\sigma)) \notin \Psi\left(z_{2}\right)$ which is trivially true in any interpretation. Also, just as positive spiders could be deleted when their informational content was represented by negative spiders, negative spiders can be deleted when their information is provided by positive spiders, by shading or, even, by other negative spiders.

To illustrate, in figure 8 the negative spider, $\sigma$, labelled $b$ is deleted. This deletion does not weaken information since the positive spider labelled $b$ provides the same absence information, albeit in a different form: from the positive spider, we can deduce that $b$ is absent from the set represented by $(\{Q\},\{P\})$. In fact, from $d$, any one of the negative spiders can be deleted. In the case of $\bar{a}$, the zone in which it is located is shaded and contains no part of any other spider: it is an empty zone. Therefore, the shading alone tells us that $a$ does not lie in the set represented by $(\{P\},\{Q\})$, so deleting $\bar{a}$ loses no information. In the remaining case, there are two $\bar{c}$ s occupying the same zone and either one of them (but not both) can be deleted whilst preserving the informational content of $d$.

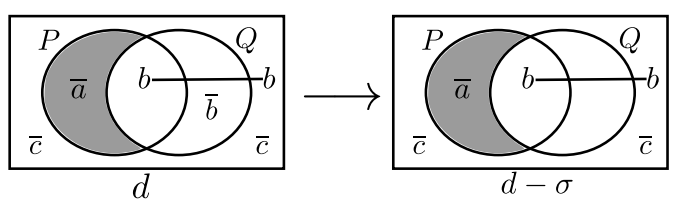

Figure 8. Delete negative spider.

Inference Rule 4 (Delete Negative Spider). Let $d=$ $(L, Z, S h Z, E S, P S, N S, \eta, \rho)$ be a consistent enhanced spider diagram. Let $\sigma$ be a negative spider in $d$. If

1. there is not a unique zone in $\eta(\sigma)$, that is

$$
|\eta(\sigma)| \neq 1
$$

or

2. there is a unique zone, $z$, in $\eta(\sigma)$, so $|\eta(\sigma)|=1$, where either

(a) the zone $z$ is empty, that is $z \in E Z(d)$,

(b) there exists a positive spider, $\sigma^{\prime}$, where $\rho\left(\sigma^{\prime}\right)=$ $\rho(\sigma)$, whose location does not include $z$, that is $z \notin \eta\left(\sigma^{\prime}\right)$, or

(c) there exists a negative spider, $\sigma^{\prime}$, where $\rho\left(\sigma^{\prime}\right)=$ $\rho(\sigma)$, whose location is $z$, that is $\{z\}=\eta\left(\sigma^{\prime}\right)$

then $d$ may be replaced by $d-\sigma$.

Lemma 9. Delete negative spider is sound and results in a semantically equivalent diagram.

Proof. Firstly, it is trivial that deleting a negative spider is sound. Therefore we focus on shown that $d-\sigma$ semantically entails $d$. There are two cases, reflecting the definition of this inference rule: either (i) $\eta(\sigma)$ contains at least two zones or (ii) it contains a unique zone. In either case, we start assuming that $I=(U, \psi, \Psi)$ is a model for $d-\sigma$ with valid $\psi^{\prime}: E S \cup P S \cup(N S \backslash\{\sigma\}) \rightarrow U$. We need to show that 
$I$ is a model for $d$. We extend $\psi^{\prime}$ to $\psi^{\prime \prime}: E S \cup P S \cup N S \rightarrow U$ where $\psi^{\prime \prime}$ is identical to $\psi^{\prime}$ except that $\psi^{\prime \prime}(\sigma)$ is defined to be $\psi(\rho(\sigma))$. In either case (i) or (ii), it is trivial that all conditions for $I$ to be a model hold for $d$ except for the negative spiders condition.

Our task is to show that this condition holds. In the case (i) where $\eta(\sigma)$ contains at least two zones, it is trivial that there is a zone, $z$, in $\eta(\sigma)$ where $\psi^{\prime \prime}(\sigma) \notin \Psi(z)$ and we are done. In case (ii), $\eta(\sigma)$ contains exactly one zone. We show $\psi^{\prime \prime}(\sigma) \notin \eta(\sigma)$. There are three sub-cases, as in the definition of the delete negative spider inference rule.

(a) In this case, the zone, $z$, in $\eta(\sigma)$ is in $E Z(d)$. In this case, $\Psi(z)=\Psi(\eta(\sigma))=\emptyset$, by lemma 2. Hence $\psi^{\prime \prime}(\sigma) \notin \Psi(\eta(\sigma))$ as required.

(b) Assume now that there is a positive spider, $\sigma^{\prime}$, where $\rho\left(\sigma^{\prime}\right)=\rho(\sigma)$. In which case, we know from the definition of the inference rule that $\eta\left(\sigma^{\prime}\right)$ does not include the unique zone, $z$, in $\eta(\sigma)$. From the positive spiders condition for $d-\sigma$, we know that $\psi^{\prime}\left(\sigma^{\prime}\right) \in \Psi\left(\eta\left(\sigma^{\prime}\right)\right)$. Since distinct zones represent disjoint sets, we deduce that $\psi^{\prime}\left(\sigma^{\prime}\right) \notin \Psi(\eta(\sigma))$. Since $\psi^{\prime \prime}\left(\sigma^{\prime}\right)=\psi^{\prime}\left(\sigma^{\prime}\right)=$ $\psi\left(\rho\left(\sigma^{\prime}\right)\right), \rho\left(\sigma^{\prime}\right)=\rho(\sigma)$, and $\psi^{\prime \prime}(\sigma)=\psi(\rho(\sigma))$, it follows that $\psi^{\prime \prime}(\sigma) \notin \Psi(\eta(\sigma))$ as required.

(c) Finally, we consider the case where there exists a negative spider, $\sigma^{\prime}$, where $\rho\left(\sigma^{\prime}\right)=\rho(\sigma)$. In this case, we know that $\eta\left(\sigma^{\prime}\right)$ contains precisely the zone, $z$, in $\eta(\sigma)$. By lemma 3, it follows that

$$
\psi\left(\rho\left(\sigma^{\prime}\right)\right)=\psi(\rho(\sigma)) \notin \Psi(z) .
$$

By definition we have $\psi^{\prime \prime}(\sigma)=\psi(\rho(\sigma))$, so

$$
\psi^{\prime \prime}(\sigma) \notin \Psi(z)
$$

as required.

Since $\psi^{\prime \prime}$ is otherwise identical to $\psi^{\prime}$ and the negative spiders condition holds for $d-\sigma$, the negative spiders condition holds for $d$. Hence $I$ models $d$ and it follows that the delete negative spiders inference rule is sound and produces a semantically equivalent diagram.

Another way to reduce clutter arising from spiders is to remove zones from their locations. Focusing first on existential spiders, sometimes their locations can be shrunk when we have information provided by negative spiders. However, we can never remove shaded zones from their locations, as this would reduce the upper bound placed on the cardinality of the associated set and, thus, not be sound. Again, when defining this rule we must be mindful of the fact that negative spiders do not provide distinctness information: carelessly removing a zone from an existential spider's location could reduce the associated lower bound on set cardinality and would not result in an equivalent diagram.

Figure 9 illustrates how we can shrink an existential spider. Here, in $d$ we can see that $c$ must lie in the set represented by $(\{P, Q\}, \emptyset)$, due to the negative zones for $c$ and the fact that $c$ cannot be in $(\{P\},\{Q\})$ due to the shading (this shaded zone is empty). Therefore, we can shrink the existential spider, removing the zone $(\{Q\},\{P\})$ from its location without weakening information.

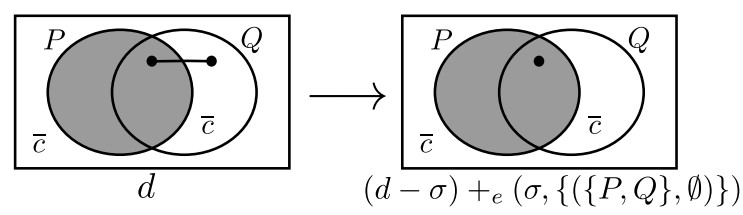

Figure 9. Shrink existential spider.

Inference Rule 5 (Shrink Existential Spider). Let $d=$ $(L, Z, S h Z, E S, P S, N S, \eta, \rho)$ be a consistent enhanced spider diagram. Let $\sigma$ be an existential spider in d occupying at least two zones of which one, $z$, is not shaded. If

1. there are no other existential or positive spiders in $d$, whose location overlaps with $\sigma$ 's, that is for all $\sigma^{\prime}$ in $(E S \cup P S) \backslash\{\sigma\}, \eta(\sigma) \cap \eta\left(\sigma^{\prime}\right)=\emptyset$, and

2. some constant, $c$, must represent an individual in the set denoted by $\eta(\sigma) \backslash\{z\}$, that is

$$
(Z \backslash E Z) \backslash N Z(c, d) \subseteq \eta(\sigma) \backslash\{z\} .
$$

Then d may be replaced by $(d-\sigma)+{ }_{e}(\sigma, \eta(\sigma) \backslash\{z\})$.

Lemma 10. Shrink existential spider is sound and results in a semantically equivalent diagram.

Proof. It is trivial to show that $d-\sigma+_{e}(\sigma, \eta(\sigma) \backslash\{z\})$ semantically entails $d$. We show that $d$ semantically entails $d-\sigma+_{e}(\sigma, \eta(\sigma) \backslash\{z\})$. Let $I=(U, \psi, \Psi)$ be a model for $d$ with valid $\psi^{\prime}: E S \cup P S \cup N S \rightarrow U$. We need to show that $I$ is a model for $d-\sigma+_{e}(\sigma, \eta(\sigma) \backslash\{z\})$. It is trivial that the missing zones condition holds for $d-\sigma+_{e}(\sigma, \eta(\sigma) \backslash\{z\})$ since it holds for $d$. We focus on the remaining five conditions. There are two cases to consider, based on the zone, $z^{\prime}$, in which $\psi^{\prime}(\sigma)$ represents an element. The first case is where $z^{\prime} \neq z$ and the second case is where $z^{\prime}=z$.

For the first case, we show that $\psi^{\prime}$ is valid for $d-\sigma+e$ $(\sigma, \eta(\sigma) \backslash\{z\})$. It is trivial that the shaded zones condition, spider distinctness condition, positive spiders condition and negative spiders condition hold for $d-\sigma+_{e}(\sigma, \eta(\sigma) \backslash\{z\})$ under $\psi^{\prime}$ since the conditions remain unchanged from those for $d$. What remains is to show that the existential spiders condition holds and here we only need to worry about $\sigma$ since all other spider locations remain unchanged. By the existential spiders condition for $d$, we also know that

$$
\psi^{\prime}(\sigma) \in \Psi(\eta(\sigma))
$$


so, since $z^{\prime} \neq z$,

$$
\psi^{\prime}(\sigma) \in \Psi(\eta(\sigma)) \backslash \Psi(z) .
$$

Therefore

$$
\psi^{\prime}(\sigma) \in \Psi(\eta(\sigma) \backslash\{z\})
$$

as required. Therefore when $z^{\prime} \neq z, I$ is a model for $d-$ $\sigma+_{e}(\sigma, \eta(\sigma) \backslash\{z\})$.

We must now consider the case where $z^{\prime}=z$. Here we must define an alternative mapping of spiders to elements, since $\psi^{\prime}$ will not ensure $\psi^{\prime}(\sigma)$ represents an element in $\Psi(\eta(\sigma) \backslash\{z\})$. Noting, by the definition of the shrink existential spider rule, that $c$ is a constant where

$$
(Z \backslash E Z) \backslash N Z(c, d) \subseteq \eta(\sigma) \backslash\{z\}
$$

we define $\psi^{\prime \prime}: E S \cup P S \cup N S \rightarrow U$ by

$$
\psi^{\prime \prime}\left(\sigma^{\prime}\right)= \begin{cases}\psi^{\prime}\left(\sigma^{\prime}\right) & \text { if } \sigma^{\prime} \neq \sigma \\ \psi(c) & \text { otherwise. }\end{cases}
$$

We now show that $I$ is a model for $d-\sigma+_{e}(\sigma, \eta(\sigma) \backslash\{z\})$ using $\psi^{\prime \prime}$.

For the shading condition, we need to verify, for each shaded zone, $z^{\prime \prime}$, in $S h Z$ that

$$
\Psi\left(z^{\prime \prime}\right) \subseteq\left\{\psi^{\prime \prime}\left(\sigma^{\prime}\right): \sigma^{\prime} \in E S \cup P S\right\} .
$$

It can readily be shown that

$$
\Psi\left(z^{\prime \prime}\right) \subseteq\left\{\psi^{\prime}\left(\sigma^{\prime}\right): \sigma^{\prime} \in E S \cup P S\right\} \backslash\left\{\psi^{\prime}(\sigma)\right\}
$$

because $\psi^{\prime}(\sigma) \in \Psi(z), z \neq z^{\prime \prime}$ and so $\psi^{\prime}(\sigma) \notin \Psi\left(z^{\prime \prime}\right)$. Therefore

$$
\Psi\left(z^{\prime \prime}\right) \subseteq\left\{\psi^{\prime \prime}\left(\sigma^{\prime}\right): \sigma^{\prime} \in E S \cup P S\right\} \backslash\left\{\psi^{\prime}(\sigma)\right\}
$$

so

$$
\Psi\left(z^{\prime \prime}\right) \subseteq\left\{\psi^{\prime \prime}\left(\sigma^{\prime}\right): \sigma^{\prime} \in(E S \backslash\{\sigma\}) \cup P S\right\} .
$$

Therefore

$$
\begin{aligned}
\Psi\left(z^{\prime \prime}\right) & \subseteq\left\{\psi^{\prime \prime}\left(\sigma^{\prime}\right): \sigma^{\prime} \in(E S \backslash\{\sigma\}) \cup P S\right\} \cup\left\{\psi^{\prime \prime}(\sigma)\right\} \\
& =\left\{\psi^{\prime \prime}\left(\sigma^{\prime}\right): \sigma^{\prime} \in E S \cup P S\right\}
\end{aligned}
$$

as required. Therefore the shaded zones condition holds.

For the spider distinctness condition, let $\sigma_{1}$ and $\sigma_{2}$ be spiders in $E S \cup P S$. If neither $\sigma_{1}$ nor $\sigma_{2}$ are the spider $\sigma$ then it follows, by the spider distinctness condition for $d$, that

$$
\psi^{\prime \prime}\left(\sigma_{1}\right)=\psi^{\prime \prime}\left(\sigma_{2}\right) \Rightarrow \sigma_{1}=\sigma_{2} .
$$

Suppose, without loss of generality, that $\sigma_{1}=\sigma$. We must show that if $\psi^{\prime \prime}(\sigma)=\psi^{\prime \prime}\left(\sigma_{2}\right)$ then $\sigma=\sigma_{2}$. Assuming $\psi^{\prime \prime}(\sigma)=\psi^{\prime \prime}\left(\sigma_{2}\right)$, we have $\psi^{\prime \prime}(\sigma)=\psi(c)=\psi^{\prime \prime}\left(\sigma_{2}\right)=$ $\psi^{\prime}\left(\sigma_{2}\right)$. Consider $\psi(c)$. Since the missing zones condition holds, by lemma $4, \psi(c) \in \Psi(Z)$. By lemma 2 , it follows that

$$
\psi(c) \in \Psi(Z \backslash E Z) .
$$

By lemma 3, we further deduce that

$\psi(c) \in \Psi(Z \backslash E Z) \backslash \Psi(N Z(c, d))=\Psi((Z \backslash E Z) \backslash N Z(c, d))$.

By the definition of the shrink existential spider rule,

$$
N Z(c, d)=(Z \backslash E Z) \backslash N Z(c, d) \subseteq \eta(\sigma) \backslash\{z\},
$$

so

$$
\psi(c) \in \Psi(\eta(\sigma) \backslash\{z\}) \subseteq \Psi(\eta(\sigma)) .
$$

We also have, by the existential and positive spiders condition for $d$

$$
\psi(c)=\psi^{\prime \prime}\left(\sigma_{2}\right)=\psi^{\prime}\left(\sigma_{2}\right) \in \Psi\left(\eta\left(\sigma_{2}\right)\right) .
$$

Therefore, since distinct zones represent disjoint sets,

$$
\eta(\sigma) \cap \eta\left(\sigma_{2}\right) \neq \emptyset .
$$

By the definition of the shrink existential spider rule, no positive or existential spider has a location that overlaps with $\eta(\sigma)$, other than $\sigma$ itself. Therefore, $\sigma=\sigma_{2}$ as required. Hence the spiders distinctness condition holds for $d-\sigma+_{e}(\sigma, \eta(\sigma) \backslash\{z\})$.

Focusing now on the existential spiders condition for $d-$ $\sigma+_{e}(\sigma, \eta(\sigma) \backslash\{z\})$, the only way this can fail is if $\psi^{\prime \prime}(\sigma)=$ $\psi(c)$ is not in $\Psi(\eta(\sigma))$. We have just seen that

$$
\psi(c) \in \Psi(\eta(\sigma) \backslash\{z\}) \subseteq \Psi(\eta(\sigma))
$$

so it follows that the existential spiders condition holds for $d-\sigma+_{e}(\sigma, \eta(\sigma) \backslash\{z\})$. It is trivial that the positive and negative spiders conditions hold, since they are identical for $d$ and $d-\sigma+_{e}(\sigma, \eta(\sigma) \backslash\{z\})$. Hence $I$ is a model for $d$. It follows that the shrink existential spiders rule is sound and produces a semantically equivalent diagram.

In the above rule, we know that the set represented by $\eta(\sigma) \backslash\{z\}$ must contain $\psi(c)$ so it is not empty. It is therefore possible to shrink $\sigma$, removing $z$, without weakening information, in part since $z$ is not shaded and in part since no other existential or positive spider has a location that overlaps with $\sigma$. We can also shrink positive spiders, when their locations include a negative zone.

Inference Rule 6 (Shrink Positive Spider). Let $d=$ $(L, Z, S h Z, E S, P S, N S, \eta, \rho)$ be a consistent enhanced spider diagram. Let $\sigma$ be a positive spider in d occupying at least two zones where $\eta(\sigma) \cap N Z(\rho(\sigma), d) \neq \emptyset$. Let $z \in \eta(\sigma) \cap N Z(\rho(\sigma), d)$. Then $d$ may be replaced by $(d-\sigma)+{ }_{p}(\sigma, \eta(\sigma) \backslash\{z\}, \rho(\sigma))$ and vice versa. 
Lemma 11. Shrink positive spider is sound and results in a semantically equivalent diagram.

Proof Sketch. It is trivial to show that $d-\sigma+_{p}$ $(\sigma, \eta(\sigma) \backslash\{z\}, \rho(\sigma))$ semantically entails $d$. We show that $d$ semantically entails $d-\sigma+_{p}(\sigma, \eta(\sigma) \backslash\{z\}, \rho(\sigma))$. Let $I=(U, \psi, \Psi)$ be a model for $d$ with valid $\psi^{\prime}: E S \cup P S \cup$ $N S \rightarrow U$. We need to show that $I$ is a model for $d-$ $\sigma+_{p}(\sigma, \eta(\sigma) \backslash\{z\}, \rho(\sigma))$. In this case, it is straightforward to show that $\psi^{\prime}$ is valid for $d-\sigma+_{p}(\sigma, \eta(\sigma) \backslash\{z\}, \rho(\sigma))$, using lemma 3 to verify that the positive spiders condition holds.

It is also possible to define a rule for shrinking negative spiders (when they include at least three zones in their locations). However, we have already seen that negative spiders with multiple zone locations can be deleted without losing information. Therefore we do not need a shrink negative spider inference rule in order to explore clutter reduction.

Lastly, we consider when it is possible to swap between different types of spider. Sometimes it is sound to swap an existential spider for a positive spider with the same location but this has no material impact on clutter so we omit this case. It is not sound to swap an existential spider, $\sigma$, for negative spiders as this would either reduce the lower bound on the set denoted by the location of $\sigma$ or introduce new information about some specific individual. Therefore, there is only one interesting case where we can swap between types of spider: swapping between positive and negative spiders can alter the visual clutter in enhanced spider diagrams.

Swapping spiders is illustrated in figure 10 , where the positive spider, $\sigma$, namely $a-a-a$, is swapped for two negative spiders, $\sigma_{1}$ and $\sigma_{2}$. These negative spiders occupy the two non-empty (single zone) regions $r_{1}=$ $\{(\{Q, R\},\{P\})\}$ and $r_{2}=\{(\{Q\},\{P, R\})\}$ that did not previously contain negative $a$ spiders. It is clear to see the information that $a$ is in the set $U \backslash(Q \cup R)$ is not lost when this swap is performed.

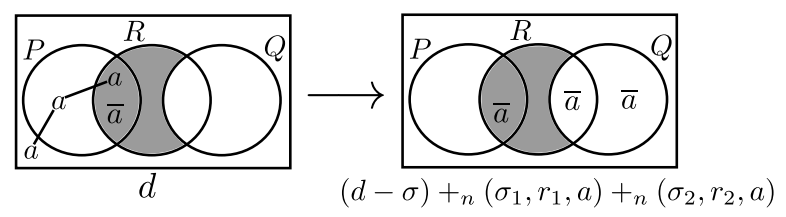

Figure 10. Swapping spiders.

Inference Rule 7 (Swap Positive and Negative Spiders). Let $d=(L, Z, S h Z, E S, P S, N S, \eta, \rho)$ be a consistent enhanced spider diagram. Let $\sigma$ be a positive spider in $d$ where
1. there are no other existential or positive spiders in d, whose location overlaps with $\sigma^{\prime}$ 's, that is for all $\sigma^{\prime}$ in $(E S \cup P S) \backslash\{\sigma\}, \eta(\sigma) \cap \eta\left(\sigma^{\prime}\right)=\emptyset$, and

2. the only shaded zones in $\sigma$ 's location are in $N Z(\rho(\sigma), d)$, that is

$$
\eta(\sigma) \cap S h Z \subseteq N Z(\rho(\sigma), d) .
$$

Let $\sigma_{1}, \ldots, \sigma_{m}$ be $m$ fresh spiders, one for each zone in

$$
Z \backslash(\eta(\sigma) \cup N Z(\rho(\sigma), d) \cup E Z)=\left\{z_{1}, \ldots, z_{m}\right\} .
$$

Then $d$ may be replaced by

$$
(d-\sigma)+{ }_{n}\left(\sigma_{1},\left\{z_{1}\right\}, \rho(\sigma)\right)+{ }_{n} \ldots .+{ }_{n}\left(\sigma_{m},\left\{z_{m}\right\}, \rho(\sigma)\right)
$$

and vice versa.

Lemma 12. Swapping positive and negative spiders is sound and results in a semantically equivalent diagram.

Proof Sketch. Firstly, it is straightforward to show that

$$
d+_{n}\left(\sigma_{1},\left\{z_{1}\right\}, \rho(\sigma)\right)+{ }_{n} \ldots .+{ }_{n}\left(\sigma_{m},\left\{z_{m}\right\}, \rho(\sigma)\right)
$$

is semantically equivalent to $d$; intuitively, negative spiders are added to non-empty zones that are not in the location of $\sigma$. The spider $\sigma$ can be deleted from

$$
d+_{n}\left(\sigma_{1},\left\{z_{1}\right\}, \rho(\sigma)\right)+{ }_{n} \ldots .+{ }_{n}\left(\sigma_{m},\left\{z_{m}\right\}, \rho(\sigma),\right.
$$

using the delete positive spider rule to give, by lemma 8 , the semantically equivalent diagram,

$$
d+_{n}\left(\sigma_{1},\left\{z_{1}\right\}, \rho(\sigma)\right)+{ }_{n} \ldots{ }_{n}\left(\sigma_{m},\left\{z_{m}\right\}, \rho(\sigma)-\sigma,\right.
$$

. Noting the commutativity of the transformations, this diagram is

$$
(d-\sigma)+{ }_{n}\left(\sigma_{1},\left\{z_{1}\right\}, \rho(\sigma)\right)+{ }_{n} \ldots .+{ }_{n}\left(\sigma_{m},\left\{z_{m}\right\}, \rho(\sigma)\right) .
$$

Therefore, swapping positive and negative spiders is sound and results in a semantically equivalent diagram.

The lemmas in this section combine to establish that all of the inference rules are sound:

Theorem 3. The inference rules are all sound and result in semantically equivalent diagrams.

\section{Measuring and Reducing Clutter}

The clutter measure given in [3] readily generalizes to enhanced spider diagrams. At the drawn diagram level, the measure counts the number of nodes and the lines used to connect spider nodes ${ }^{2}$. For each spider, the number of lines

\footnotetext{
${ }^{2}$ Recall that this measure of clutter was empirically evaluated in [16] where it was found that high levels of clutter resulted in worse task performance.
} 
is one less than the number of zones in its location. For example, in figure $2, d_{3}$ has a clutter score of 13 , since there are seven nodes and six connecting lines, whereas $d_{4}$ has a score of 1 . In figure $4, d_{7}$ has a score of 0 but the semantically equivalent diagram, $d_{8}$, has a score of 11 .

Definition 11. Let $d=(L, Z, S h Z, E S, P S, N S, \eta, \rho)$ be an enhanced spider diagram. The spider clutter score for $d$, denoted $\mathcal{S C S}(d)$, is

$$
\mathcal{S C S}(d)=\sum_{\sigma \in S(d)}(2|\eta(\sigma)|-1)
$$

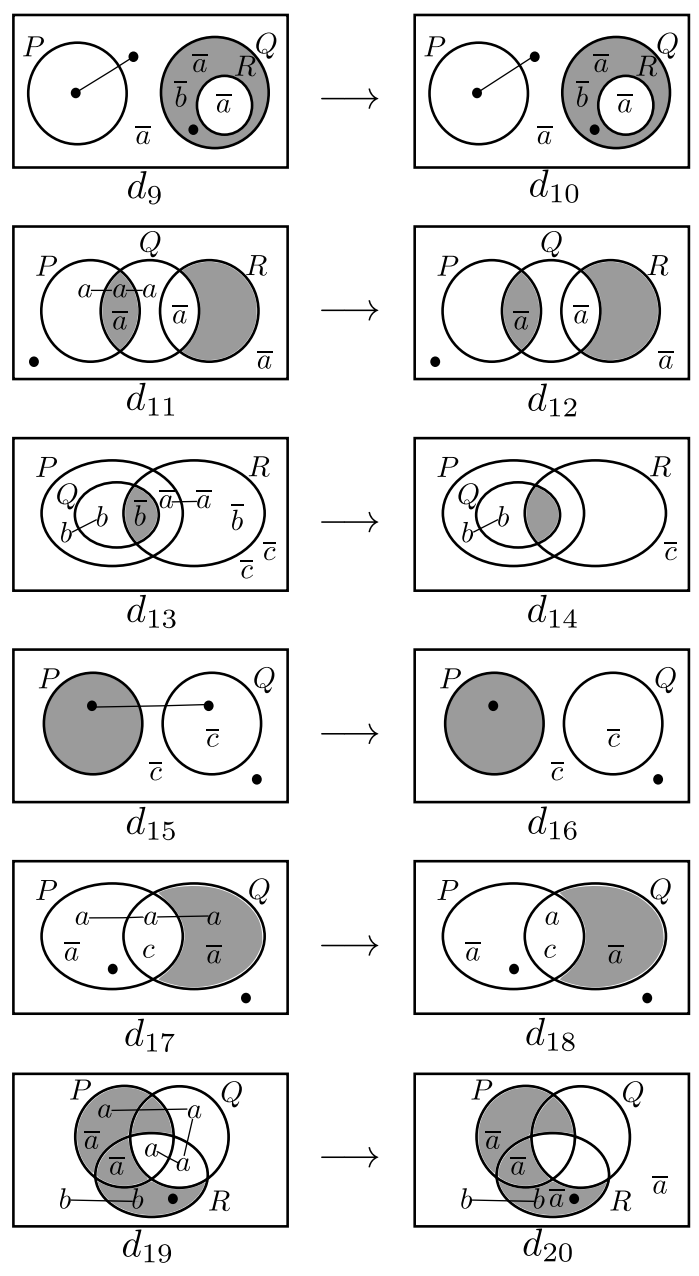

Figure 11. Rules' impact the clutter score.

We now demonstrate how the rules can impact the clutter score. In figure 11, the first three rows illustrate the three deletion rules. Deleting the existential spider located in two zones in $d_{9}$ to give $d_{10}$ reduces the score by 3 . This spider can only be deleted, without losing information, since the negative spiders express that $a$ is absent from $Q$. The region outside $Q$ therefore 'contains' $a$ and it is precisely this region that contains the existential spider that is deleted to give $d_{10}$. Deleting the positive spider, $a$, from $d_{11}$ to give $d_{12}$ reduces the clutter score by 5 . This time, the information provided about the individual $a$ by the positive spider can be inferred from the negative spiders, $\bar{a}$, and so $a$ is redundant. These two rules clearly show that absence information, provided by negative spiders, leads to a reduction in visual clutter. When it comes to deleting negative spiders, there are various cases illustrated in $d_{13}$ and $d_{14}$. Deleting $\bar{a}-\bar{a}$ reduces the score by 3 , deleting two $\bar{b}$ s by 2 and one $\bar{c}$ by 1 (total: 6 ).

The next two rows show applications of shrinking rules. Without absence information, it is not possible to shrink spiders and maintain the semantic information. In $d_{15}$, we can infer that $c \in P \backslash Q$, since $c \notin Q \backslash P$ and $c \notin \overline{P \cup Q}$. Thus, the existential spider in $d_{15}$ must represent the same element as $c$, and be in $P$. Therefore we can remove a zone from its location, as shown in $d_{16}$, reducing the score by 2. The case for $d_{17}$ is more straightforward: the absence information about $a$ allows us to reduce the location of the $a-a-a$ spider, lowering the clutter score by 4 after two applications of the shrink positive spider rule.

Lemma 13. A single application of any one of the three deletion rules reduces the clutter score by $2|\eta(\sigma)|-1$ where $\sigma$ is the deleted spider.

Lemma 14. A single application of any one of the two shrinking rules reduces the clutter score by 2 where $\sigma$ is the shrunk spider.

The last row of figure 11 shows an application of the swap rule. The $a-a-a-a$ spider is swapped for two $\bar{a}$ spiders, reducing the clutter score by 5 .

Lemma 15. A single application of the swap rule reduces clutter whenever

$$
2|\eta(\sigma)|-1>|Z \backslash(\eta(\sigma) \cup N Z(\rho(\sigma), d) \cup E Z)|,
$$

where $\sigma$ is the positive spider to be swapped.

\section{Discussion on Clutter Reduction}

We now discuss how clutter reduction using absence, conveyed via negative spiders, contrasts with the Venn- $\mathrm{i}^{\mathrm{e}}$ case. In enhanced spider diagrams, many of the rules that we have introduced required the subject existential or positive spider, $\sigma$, to be the only one in its location. This is because to delete, shrink or swap such a spider, absence information must be used, yet negative spiders do not provide distinctness information. Considering an absence spider in isolation, it only indicates the set in which the represented individual does not lie and, therefore, in which set it does lie. Of course, this is identical for $\bar{i}$-sequences in 
Venn- $\mathrm{i}^{\mathrm{e}}$. However, a significant difference is that, in Venn$\mathrm{i}^{\mathrm{e}}, \otimes$-sequences and $i$-sequences do not represent distinct elements, so Venn-i $\mathrm{i}^{\mathrm{e}}$ 's inference rules are much less restrictive.

An example can be seen in figure 12. Firstly, we note that $D$ uses an $\otimes$-sequence, which asserts that the set $R \backslash P$ is not empty, as well as $i$-sequences (for presence) and $\bar{i}$ sequences (for absence). To create $D_{1}$, the $\bar{a}$-sequences are swapped for an $a$-sequence, a transformation which in general can increase clutter. This results in a diagram where all information about the sets in which particular individuals lie is given using presence information. The diagram $D_{2}$ is created by iteratively shrinking $a$-sequences, as we can deduce from $D_{1}$ that $a$ is in the set $R \cap \bar{P} \cap \bar{Q}$. The next step shrinks the $\otimes$-sequence: in Venn-i 's, shaded regions always represent the empty set, so we can remove the $\otimes$ symbol from the shaded zone, shrinking the sequence. Next, we notice that, since $a$ represents an individual in the set $R \cap \bar{P} \cap \bar{Q}$, the $\otimes$-sequence is redundant (here, it is important to recall that distinct sequences do not denote distinct individuals in Venn-i $\mathrm{i}^{\mathrm{e}}$. Lastly, we can swap $b--b--b$ for a single $\bar{b}$, giving us $D_{\min }$. The diagram $D_{\min }$ is semantically equivalent to $D$ and is minimally cluttered. It should be evident from this example that not asserting the distinctness of individuals has led to more freedom in when inference rules can be applied than we saw for enhanced spider diagrams, albeit at the expense of expressive power.
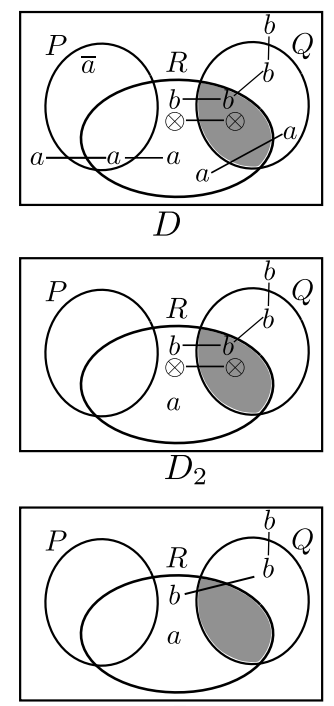

$D_{4}$
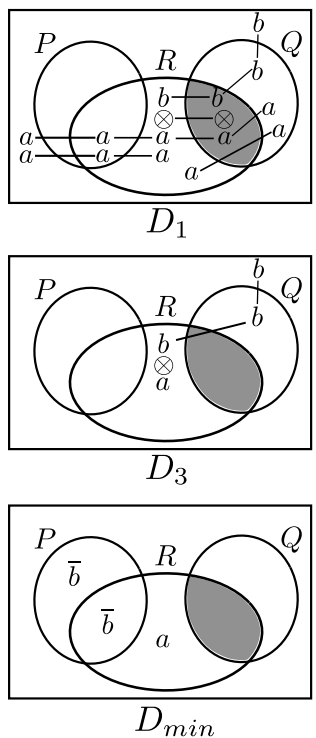

Figure 12. Rules' impact the clutter score.

In summary, as a consequence of the key differences between Venn- $\mathrm{i}^{\mathrm{e}}$ and enhanced spider diagrams, there are many more situations in Venn- $\mathrm{i}^{\mathrm{e}}$ in which information about the absence of individuals can be used to reduce clutter. A less diagrammatic interpretation of absence, whereby nega- tive spiders $d o$ assert that the represented individual is distinct from those represented by the other spiders, would lead to the ability to reduce clutter further. This will be an interesting avenue for future work, particularly with regard to the usability of the resulting logic relative to enhanced spider diagrams.

\section{Conclusion}

We have introduced enhanced spider diagrams, which include syntax for visually representing the absence of individuals from particular sets directly. Necessary and sufficient conditions for diagram satisfiability were given, since unsatisfiable diagrams are semantically equivalent to diagrams with a zero clutter score. Following from this, we defined inference rules that permitted clutter to be reduced in satisfiable diagrams. Interestingly, the level of clutter reduction that is possible in this system is not as dramatic as was seen for the Venn- $\mathrm{i}^{\mathrm{e}}$ system, where $i$-sequences and $\bar{i}$-sequences could readily be swapped to alter clutter. As indicated, an alternative, less well-matched, interpretation of negative spiders could lead to larger reductions in visual clutter. However, it is unclear whether lower clutter, with a less well-matched syntax, or higher clutter with a wellmatched syntax is most effective for human cognition. Such a trade-off should be explored as it could provide important insight into choices that must be made when designing diagrammatic systems. Future work should also seek to understand the impact of clutter reduction on user task performance. Both of these aspects are important for ensuring an effective system of logic, accessible to users of the notation, is produced.

Acknowledgement This research was conducted whilst Gem Stapleton was Visiting Professor at Jadavpur University and is partially funded by a Leverhulme Trust Research Project Grant (RPG-2016-082) for the project entitled Accessible Reasoning with Diagrams.

\section{References}

[1] M. Alqadah, G. Stapleton, J. Howse, and P. Chapman. Evaluating the impact of clutter in Euler diagrams. In 8th Int. Conf. on Diagrams, pages 109-123. Springer, 2014.

[2] R. Bhattacharjee, M. Chakraborty, and L. Choudhury. Venn diagram with names of individuals and their absence: A nonclassical diagram logic. Logica Universalis, pages 1-66, 2018.

[3] J. Burton, M. Chakraborty, L. Choudhury, and G. Stapleton. Minimizing clutter using absence in Venn- $\mathrm{i}^{\mathrm{e}}$. In 9th Int. Conf. on Diagrams, pages 107-122. Springer, 2016.

[4] L. Choudhury and M. K. Chakraborty. On extending Venn diagrams by augmenting names of individuals. In 3 rd Int. Conf. on Diagrams, pages 142-146. Springer, 2004. 
[5] J. Gil, J. Howse, and S. Kent. Formalising spider diagrams. In IEEE Symposium on Visual Languages (VL99), Tokyo, pages 130-137. 1999.

[6] C. Gurr. Effective diagrammatic communication: Syntactic, semantic and pragmatic issues. J. Visual Languages and Computing, 10(4):317-342, 1999.

[7] L. Horn. A Natural History of Negation. CSLI Lecture Notes. Center for the Study of Language and Information, 2001.

[8] T. Hou, P. Chapman, and A. Blake. Antipattern comprehension: An empirical evaluation. In 9th Int. Conf. on Formal Ontology in Information Systems, pages 211-224, 2016.

[9] T. Hou, P. Chapman, and I. Oliver. Measuring perceived clutter in concept diagrams. In IEEE Symposium on Visual Languages and Human-Centric Computing, pages 31-39. 2016.

[10] J. Howse, G. Stapleton, and J. Taylor. Spider diagrams. LMS J. Computation and Mathematics, 8:145-194, 2005.

[11] J. Howse, G. Stapleton, K. Taylor, and P. Chapman. Visualizing ontologies: A case study. In International Semantic Web Conference, pages 257-272. Springer, 2011.

[12] C. John, A. Fish, J. Howse, and J. Taylor. Exploring the notion of clutter in Euler diagrams. In 4th Int. Conf. on Diagrams, pages 267-282, 2006. Springer.

[13] C. Peirce. Collected Papers, volume 4. Harvard University Press, 1933.

[14] Y. Sato, K. Mineshima, and R. Takemura. The Efficacy of Euler and Venn Diagrams in Deductive Reasoning: Empirical Findings. In 6th Int. Conf. on Diagrams, pages 6-22. Springer, 2010.

[15] S.-J. Shin. The Logical Status of Diagrams. Cambridge University Press, 1994.

[16] G. Stapleton, A. Blake, J. Burton, and A. Touloumis. Presence and absence of individuals in diagrammatic logics: An empirical comparison. Studia Logica, 105(4):787-815, 2017.

[17] G. Stapleton, L. Choudhury, and M. Chakraborty. Spider diagrams with absence. In DMS VIVA 2018. KSI, 2018.

[18] G. Stapleton, J. Taylor, J. Howse, and S. Thompson. The expressiveness of spider diagrams augmented with constants. J. Visual Languages and Computing, 20:30-49, 2009.

[19] N. Swoboda and G. Allwein. Using DAG transformations to verify Euler/Venn homogeneous and Euler/Venn FOL heterogeneous rules of inference. J. Software and System Modeling, 3(2):136-149, 2004. 